Utah State University

DigitalCommons@USU

6-1-1918

\title{
Lectotypes of the Species of Hymenoptera (Except Apoidea) Described by Abbé Provancher
}

\author{
A. B. Gahan \\ Bureau of Entomology, Washington, D.C.
}

S. A. Rohwer

Bureau of Entomology, Washington, D.C.

Follow this and additional works at: https://digitalcommons.usu.edu/bee_lab_ga

Part of the Entomology Commons

\section{Recommended Citation}

Gahan, A. B. and Rohwer, S. A., "Lectotypes of the Species of Hymenoptera (Except Apoidea) Described by Abbé Provancher" (1918). Ga. Paper 152.

https://digitalcommons.usu.edu/bee_lab_ga/152

This Article is brought to you for free and open access by the Bee Lab at DigitalCommons@USU. It has been accepted for inclusion in $\mathrm{Ga}$ by an authorized administrator of DigitalCommons@USU. For more information, please contact digitalcommons@usu.edu.

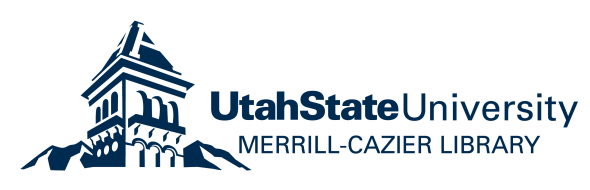




\section{3}
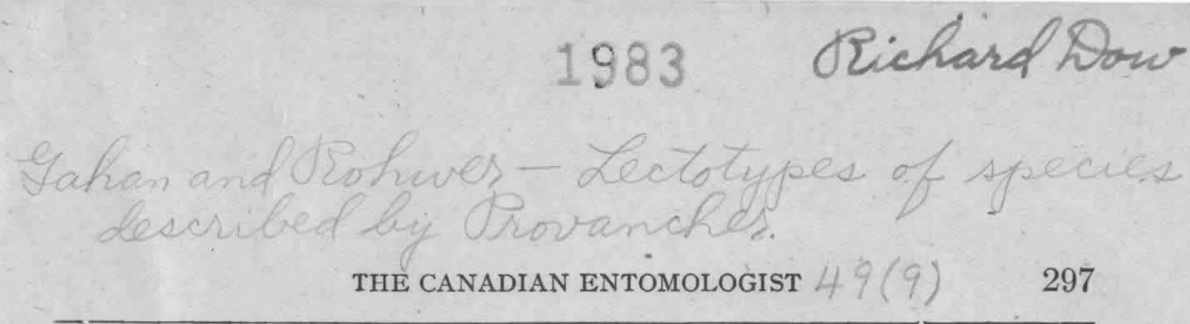

Some of the plants upon which it had bestowed its attention were later ripped from the trees by natives with long, sharp knives, and in the course of time finally came to grace the conservatory of a person whose resthetic taste demanded orchids. It was only a question of time before Cholus made her appearance and started to feed on the scenery in that conservatory. It appears that she had smuggled either herself or her young along with the plants, undoubtedly in the tissue, which the inspector could not examine without destroying the plant, and in that way arrived safely at her, destination.

Of course, the tropical atmosphere of a greenhouse does not approach that of her natural home but it is a fair substitute, and she can be contented provided the owner does not devote his attention to her extermination.

The foregoing cases, and it would be possible to cite numerous other ones, show how the insect fauna of New Jersey and other States is constantly being added to by undesirables from other countries. This immigration is taking place in spite of welldeveloped and well-enforced systems of inspection. The establishment of foreign pests in the United States is not always due to a laxity in the inspection service of any state. In most cases, the pests have entered undetected by the inspector, sometimes through his ignorance of foreign pests, sometimes on account of individual carelessness, but mostly on account of the impossibility of examining every leaf, twig, root and particle of soil around the roots of a plant and having anything left that will grow, especially when an inspector is called upon to examine hundreds of plants each day.

In other words, ordinary inspection will not keep out all foreign pests, and extraordinary inspection would not be tolerated by importing firms or paid for by state governments. The inspection does, however, prevent an overwhelming rush of such pests; it does hold and delay the spread of them at times until means of controlling them have been found or until they are no longer dangerous, and it has in the past served the country well; but it is useless to expect more from inspection however well carried out it may be.

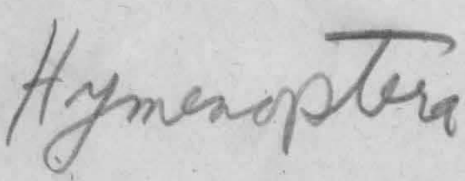

Property of
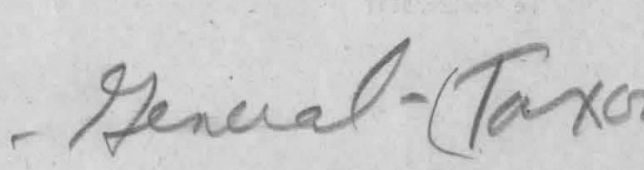
The fact remains that by the importation of plants from foreign countries $(64,652$ cases were brought into the U. S. during the season of 1915-16, according to the report of the Fed. Hort. Bd. for year ending June 30,1916 ) we are slowly but surely adding to the number of pests which we already have in this country, thereby increasing the burden which future generations will have to bear. And the remedy? A national quarantine of all foreign nursery stock.

\section{Explanation of Plate XIV.}

Fig. 1, Blaberus discoidalis, a tropical roach (natural size).

Fig. 2, Gryllotalpa gryllotalpa, the European mole cricket (natural size.)

Fig. 3, Cholus forbesii, a tropical orchid weevil, (after. H. S. Barber), (enlarged).

LECTOTYPES OF THE SPECIES OF .HYMENOPTERA (EXCEPT APOIDEA) DESCRIBED BY ABBÉ PROVANCHER.

BY A. B. GAHAN AND S. A. ROHWER, BUREAU OF ENTOMOLOGY, WASHINGTON, D.C.

\section{Introduction.}

This paper, which is a contribution from the Branch of Cereal and Forage Insects and the Branch of Forest Insects, is largely based on an examination made in May and June, 1915, of the Provancher collections located in the Museum of Public Instruction at Quebec, and in the possession of Mr. W. Hague Harrington and the Department of Agriculture at Ottawa, Canada.

This study was undertaken in order to determine in so far as possible the correct position of the Provancher species in the modern classification and obtain notes which would, supplement the original descriptions, and thus facilitate recognition of the species, many of which could not be certainly identified by the original description. Notes of greater or less extent were obtained on all but a few of the species of Hymenoptera, excluding the Apoidea, especial stress being laid, however, upon the sawflies and the parasitic forms belonging to the Ichneumonoidea. In some groups our notes consisted principally of a record of the September, 1917 
condition of the type. The limited time at our disposal made it impossible to attempt to secure full notes on any but the groups in which we were especially interested.

This paper is only a list of the species described by Provancher with the location, condition and designation of the type specimens, and is submitted at this time in order to establish definite premises on which to work and thus make it possible to publish in the future, systematic notes on the species with assurance that other workers will be able to use our remarks and find the same specimens we examined.

The only previous comprehensive study of the Provancher collections was made by Mr. G. C. Davis, who has published the results of his study in two reportst. Davis, however, confined himself entirely to the Ichneumonidæ. The conclusions reached by him regarding the species, in the main, agree with our own, but in a number of instances do not coincide with ours. Davis made no attempt to establish lectotypes for the species, and, therefore, it was often impossible for us to determine on what specimen he based his conclusion.

\section{Provancher's Life and Work.}

Practically the first Canadian and in fact one of the first Americans to make a serious and comprehensive study of the "Hymenoptera of Canada was Abbé Léon Provancher. Abbé Provancher was a French Canadian who was born, brought up and spent most of his life in the Province of Quebec. He died in 1892 , and brief accounts of his life and work were afterward published in a number of journals.* Some years later Abbé Huard began a more extended biography which appeared in various issues of Le Naturaliste Canadien, ** a magazine founded and edited, until shortly before his death, by Provancher. Since Provancher

†Some notes fróm a Study of the Provancher Collection of Ichneumonidæ, 1894, Proc. Acad. Nat. Sc. Phil., pp. 184-190.

Review of a few more Provancher types of Ichneumonidæ, 1895, Can. Ent., pp. $287-290$.

*See especially Can. Ent., Vol. 24, 1892, pp. 130-131, and Entom. News, Vol. 6 , 1895 , p. 209 , pl. IX.

**This interesting account has never been completed, but Abbé Huard told us it was his intention to complete it and we certainly hope he finds an opportunity to do so. For the parts published, see Nat. Can., 1894, 1895, 1896, 1897, 1898. 
began his work in Quebec and spent most of his life there, it is not surprising to know that a large part of his collection came from that region. But later in his life he received much material from other people so that his completed collection included species

- from many parts of Canada, the United States, Europe and some of the West Indian Islands.

Provancher described about 923 species and a few genera of Hymenoptera and most of this great number are valid. His largest and most comprehensive work on Hymenoptera is Petite Faune Entomologique du Canada and its Additions, but besides this he published several shorter papers on Hymenoptera in which new species are described. Considering the time, lack of facilities and literature, and his comparative isolation, Provancher had a very good idea of the limits of a species. The weakest point of his hymenopterological work was his conception of genera and generic limits. Even when we consider the genera he recognized, we often find that he placed the same or closely allied species in widely different genera. Hence, we find, especially in the parasites, that Provancher was often wrong in his generic placement of the species. His descriptions are accurate, and if we remember that the diagnostic characters of that period were limited mostly to colour, they are as satisfactory as those of his contemporaries.

\section{Location of Collections.}

Most of Provancher's types are in the Public Museum of Quebec, some are in the collection of Mr. W. Hague Harrington at Ottawa, a few are in the collection of the Canadian Department of Agriculture at Ottawa, while a few others were returned to Ashmead and Coquillett and are now in the United States National Museum. Some few types we were unable to locate. These may have been returned to the ccllectors.

In 1889 the College de Levis, Levis, Quebec, received a collection of insects from Provancher, and for some time it was thought to contain some of his types. Further investigation tends to prove that this collection was composed entirely of duplicates, and in certain cases these were not correctly determined.

$$
\text { A-Collection in the Public Museum at Quebec. }
$$

The collection in the Museum of Natural History, under the 
Department of Public Instruction is now housed in the Parliamentary Building in Quebec, and is cared for by Abbé V. A. Huard and his assistant. In this Museum there are really two Provancher collections. The first was purchased in 1877 by the Museum and is known as the 1877 collection (in this paper referred to as the first collection). The other came to the Museum (through purchase) after Provancher's death, and is known as the Dernière Provancher collection, (in this paper referred to as the second collection). Both of these collections are in the cabinets obtained from Provancher, and most fortunately are still left as arranged by him. Each collection contains species not represented in the other, but in cases where the species was found represented in both collections and there was nothing in the description or manuscript notes to prevent, we have chosen as lectotype a specimen from the second collection, because this was the collection retained and used by Provancher until his death, and we are inclined to believe, even though he was not a "type-worshipper," that he would retain the actual type for future reference. Specimens in both collections bear small, yellow labels on which a number is printed. These numbers are species numbers and refer to a catalogue prepared by Provancher. Each insect order in both collections begins with the number one. In the Hymenoptera, therefore, considering both collections as a unit, we often have two, usually widely different species under the same number. There appears to be no instance in which the same species occurs under the same number in both collections. The two collections differ in the style of name label. The name label for the 1877 collection is on blue paper, while that for the second collection is on white paper. which has a double red line (the outer being the heavier) around the margin.

The catalogues prepared by Provancher are in the Public Museum, and although they are little more than lists of numbers followed by names, with an occasional mention of locality, they are of some assistance in proving the way in which Provancher treated species reduced by him to synonymy.

A hasty examination of all the insects in both collections showed that they were in remarkably fine condition, considering that they are kept in wooden drawers unprotected by any repellant, 
and which are closed by glass tops which set down inside, without any overlapping flange. A careful examination of the Hymenoptera proved that they were free from pests and in good condition. At the time of our visit the Hymenoptera of the 1877 collection were in museum case 35 , and those of the second collection in the left hand column of case 46 and two drawers in the left hand column of case 49 .

That Provancher had no concrete idea of the value of types is shown by the fact that in no case (with possibly a few exceptions in later years) were his types labeled as such. Furthermore, it is apparent that when he discovered one of his species to be a synonym he often removed the name label from the type and pinned the specimen among others of the species to which he thought it belonged. In other instances, upon deciding that one of his species was synonymous with another not already represented in his collection he removed the original name label and replaced it with what he considered to be the correct one. For example, there is no specimen in his collections labeled Selandria flavicornis. After describing this species Provancher concludèd that it was the same as Selandria halcyon, and an examination of the catalogue shows under 60 the name Selandria flavicornis with the word "flavicornis" crossed out and above it written the word "halcyon." It, therefore, appears certain that the type of Selandria flavicornis stands in the collection under the name Selandria halcyon. In this case this is also proven by the fact that Provancher instead of supplying a new label just reversed the old label and wrote the name Selandria halcyon so that we find on the underside of the label for $S$. halcyon the original label for Selandria flavicornis. This one case is sufficient to show how Provancher worked. Many other similar cases could be mentioned. There are many cases, however, where there is no proof, either in the catalogue or in the labeling, that the types of some of Provancher's species which were later suppressed by him stand under the name of the species with which he considered them to be synonymous. In such cases we can only assume, from our knowledge of Provancher's methods, that this is what has taken place.

At no time while studying the collection did we remove any labels, and we were always very careful to put the specimens 
back where they came from so that the collection still stands as it was arranged by Provancher. We did not even feel justified in labeling the specimens which we believed to be the type as lectotypes, and will rely in this paper entirely on the name label and the number label for means of correctly identifying the specimens which we believe should stand as type.

\section{$B$-Harrington Collection.}

The types in the Harrington collection are in good condition, and although they usually do not bear the name label in Provancher's writing we were assured by our friend $\mathrm{Mr}$. W. H. Harrington that they were the identical specimens examined by Provancher as could easily be proven by a comparison of the number (they bear in addition to other labels a small, white square on which is written by Harrington a number) with the list as returned by Provancher.

C-Types in Collection of Canadian Department of Agriculture.

The types in the Department of Agriculture are in good condition, and have all been properly labeled as types.

$$
D \text {-Types in U.S. National Museum. }
$$

The types in the United States National Museum have all been accessioned and labeled with Museum type numbers.

\section{Plan of Paper.}

In submitting this list of the species described by Provancher and presenting notes on the location and condition of the types we have considered that it was much better to arrange them alphabetically as they appear in the final index published by Provancher as a conclusion to his two more important papers on Hymenoptera. We have chosen this method largely because there will be many more changes in generic position than those already published, and also because we do not know when we shall have an opportunity to. completely review our notes and definitely assign the species of the genera as at present understood. Practically throughout this paper the word "type" is used in the sense of "lectotype." There are, however, certain cases when there was only one specimen, and there is no doubt that it is the specimen examined by Provancher and is, therefore, certainly the type. 
At the time of our visit the type specimens of the species placed by Provancher in the Braconid subfamilies Aphidiinæ and Opiinæ were not available and the data on these were not secured. We hope, however, to secure and present it later.

Unless otherwise stated, it is to be understood that the specimens are in good condition. The letter (s) stands for the word "script." The numbers on the yellow labels are printed. Whenever possible, we chose as type the specimen which bore the name label written in Provancher's hand.

\section{An Alphabetical List of Species With Designation of Lectotypes.}

Acerota opaca. Type.-Yellow label 1381. 2nd Coll. Pub. Mus., Quebec. Two other specimens. Fair.

Acoenites canadensis. Type.-Male, yellow label 375. 2nd Coll. Pub. Mus., Quebec. Lacks antennæ, right fore wing, hind tarsi, abdomen glued on.

Acoenites flavipes. Type-Female, yellow label 1249 . 2nd Coll. Pub. Mus., Quebec.

Acordulocera saginata. Type.-Yellow label 390. 2nd Coll. Pub. Mus., Quebec. Right fore wing gone.

Acothyreus mellipes. Type.-Yellow label 1320. 2nd Coll. Pub. Mus., Quebec. Fair.

Agilips aciculatus. Type--Not seen.

Agathis femorator. Type--Female, yellow label 578. 2nd Coll. Pub. Mus., Quebec. Antennæ wanting.

Agathis nigriceps. Type--Female, yellow label 1680 . 2nd Coll. Pub. Mus., Quebec.

Agathis perforator. Type--Female, yellow label 577. 2nd Coll. Pub. Mus., Quebec.

Agathis quæsitor. Type.-Female, yellow label 576. 2nd Coll. Pub. Mus., Quebec. Lacks head.

Agathis scrutator. Type--Female, yellow label 1269. 2nd Coll. Pub. Mus., Quebec. Male allotype. Both glued on slips.

Agathis tibiator. Type.-Female, yellow label 579. 2nd Coll. Pub. Mus., Quebec. 
Agenia atrata. Allotype.-Male, blue label 125(s). Yellow label 1417. 2nd Coll. Pub. Mus., Quebec.

Agenia perfecta. Type--Male, yellow label 783. 2nd Coll. Pub. Mus., Quebec. Antennæ wanting beyond 3rd joint. Four hind tibiæ gone.

Agenia rufigastra. Type.-Female, blue label 122(s), yellow label 1419. 2nd Coll. Pub. Mus., Quebec.

Allantus cogitans. Type.-Female, yellow label 44. 2nd Coll. Pub. Mus., Quebec. Lacks right antenna. Two female paratypes. 1st Coll.

Allantus robustus. Type--Female, Harrington Coll.

Allantus rubricus. Type.-Female, Harrington Coll. Lacks right antenna beyond 3rd joint.

Alomya pulchra. Type.-Not in Pub. Mus., Quebec, unless under name Phygadeuon pubescens Prov.

Alysia astigma. Type.-Female, yellow label 1051. 2nd Coll. Pub. Mus., Quebec. Left flagellum gone and only base of right remaining.

Same specimen used as type of Aspilata astigma.

Alysia completa. Type--Female, yellow label 1166. 2nd Coll. Pub. Mus., Quebec.

Alysia fossulata. Type-Cat. No. 1970, U. S. N. M.

Alysia lucens.-Type.-Female, yellow label 909. 2nd Coll. Pub. Mus., Quebec.

Alysia nigriceps. Type.-Female, yellow label 539. 2nd Coll. Pub. Mus., Quebec. Right flagellum and extreme apex of left gone.

Alysia rubriceps. Tỹpe.-Male, yellow label 1052. 2nd Coll. Pub. Mus., Quebec: Same specimen used as type of Phanocarpa rubriceps Prov.

Alyson conicus. Type--Male, blue label 622(s), yellow label 1449. 2nd Coll. Pub. Mus., Quebec. Lacks most of left flagellum.

Alyson guignardi. Type.-Female, yellow label 1433. 2nd Coll. Pub. Mus., Quebec.

Alyson triangulifer. Type--Male, yellow label 1450. 2nd Coll. Pub. Mus., Quebec. Lacks apices of antennæ. 
Amblyopone binodosa. Type--Yellow label 948. 2nd Coll. Pub. Mus., Quebec. Fair. (Arotropus binodosa).

Amblyteles bifasciatus.- Type.-Yellow label 227. 1st Coll. Pub. Mus., Quebec.

Amblyteles borealis. Type.-Female, yellow label 1002. 2nd Coll. Pub. Mus., Quebec.

Amblyteles indistinctus. Type-Female, yellow label 185. 2nd Coll. Pub. Mus., Quebec.

Amblyteles macrocephalus. Type-Male, yellow label 1063. 2nd Coll. Pub. Mus., Quebec. Both antennæ broken, one at 1st flagellar joint, other at middle, 1 anterior, 1 median and 1 hind leg missing.

Amblyteles marginatus. Type--Female, yellow label 700, 2nd Coll. Pub. Mus., Quebec.

Amblyteles perluctuosus. Type.-Female, yellow label 172. 2nd Coll. Pub. Mus., Quebec.

Amblyteles quebecensis. Type--Yellow label 181. 2nd Coll. Pub. Mus., Quebec.

Amblyteles stadaconensis. Type.-Male, yellow label 175 . 2nd Coll. Pub. Mus., Quebec.

Amblyteles superbus. Type.-Female, Harrington Coll.

Amblyteles tetricus. Type-Female, yellow label 171. 2nd Coll. Pub. Mus., Quebec.

Anacharis marginata. Type--Yellow label 1318. 2nd Coll. Pub. Mus., Quebec. Abdomen off but on triangle below.

Anacharis pediculata. Type.-Blue label 762(s), yellow label 1317. 2nd Coll. Pub. Mus., Quebec.

Anacharis subcompressa. Type.-White label "Quebec"; yellow label 616. 2nd Coll. Pub. Mus., Quebec. Abdomen gone.

Anacrabro constrictus. Type.-Male, tag-mounted, yellow label 1690. 2nd Coll. Pub. Mus., Quebec.

Anacrabro lævis. Type.-Female, yellow label 1691. 2nd Coll. Pub. Mus., Quebec.

Andricus gibbosus. Type.-Yellow label 609. 2nd Coll. Pub. Mus., Qubeec. Head and abdomen gone. Cynips (Andricus) gibbosa-under Cynips in list. 
Aneurychus foveatus. Type.-Blue label 83; yellow label 1330. 2nd Coll. Pub. Mus., Quebec. Fair.

Aneurychus mellipes. Type--Yellow label 1331. 2nd Coll. Pub. Mus., Quebec.

Anomalon chlamidatum. Type-Female, yellow label 1217. 2nd Coll. Pub. Mus., Quebec. Left antenna missing beyond fourth joint and right hind leg at coxa.

Anomalon exile. Type.-Female, yellow label 329. 1st Coll. Pub. Mus., Quebec. Left antenna at 15 th joint, left median and both hind legs at coxæ gone; left fore wing gone and right broken at middle.

Anomalon filiforme. Type.-Female, yellow label 1218. 2nd Coll. Pub. Mus., Quebec.

Anomalon nigripennis. Type--Probably pinned under Exochilum mundum Say. 1st Coll. Pub. Mus., Quebec.

Anomalon rufulum. Type, and Allotype--Harrington Coll. Both antennæ, tarsi, except one anterior, broken and lost. Female paratype yellow label 1213, blue label 481. 2nd Coll. Pub. Mus., Quebec.

Anomalon unicolor. Type-Female, yellow label 1216. 2nd Coll. Pub. Mus., Quebec.

Apanteles acaudus. Type.-Female, yellow label 1285. 2nd Coll. Pub. Mus., Quebec.

Apanteles carpatus. Type- -Apparently destroyed. Pin bearing yellow label .592 in 2nd Coll. Pub. Mus., Quebec.

Apanteles clavatus. Type.-Female, yellow label 642 . 2nd Coll. Pub. Mus., Quebec. Badly broken and plastered up with glue.. Antennæ, one median and both hind legs gone. Specimen in U. S. N. M. labeled type not type.

Apanteles cinctus. Type.-Female, yellow label 716. 2nd Coll. Pub. Mus., Quebec. Antennæ broken near middle. Faun. 529. Add. S. 388.

Apanteles crassicornis. Type.-Female, yellow label 1269. 2nd Coll. Pub. Mus., Quebec. Antennæ and hind tarsi broken.

Apanteles femur-nigrum. Type--Male, yellow label 1578. 2nd Coll. Pub. Mus., Quebec. One antenna broken, one front and one hind leg missing. 
Apanteles longicornis. Type-Female, yellow label 1258 . 2nd Coll. Pub. Mus., Quebec.

Aphæreta auripes. Type.-Specimen in Public Mus., Quebec, bearing name label in Provancher's hand. Other data not taken. Specimen in U. S. N. M. labeled type, not type.

Aphidaria basilaris. Type.-See Introduction.

Aphidius canadensis. Type.-See Introduction.

Aphidius nigrovarius. Type--See Introduction.

Aphidius obscurus. Type.-See Introduction.

Aplomerus tibialis. Type--Female, Ent. Branch, Dept. Agr., Ottawa. Left antenna wanting beyond 5 th joint.

Arenetra quebecensis. Type.-Not in Pub. Mus., Quebec, unless under Lampronota tegularis.

Arotes superbus. Type--Not in Pub. Mus., Quebec, unless under name $A$. vicinus Cress.

Arotropus binodosus.- See Amblyopone.

Ascogaster rufipes. Type.-Female, yellow label 1254. . 2nd Coll. Pub. Mus., Quebec. Rather dirty. (This number in Prov. catalogue refers to Chelonus rugulosus Prov. There is no such species.

Aspilota astigma.- See Alysia astigma.

Atractodes autumnalis. Type--Female, yellow label 706 . 2nd Coll. Pub. Mus., Quebec.

Atractodes nigricoxus. Type--Male, yellow label 984. 2nd Coll. Pub. Mus., Quebec. Left hind tarsus broken at 2nd joint.

Atractodes nitens. Type--Male, yellow label 695. 2nd Coll. Pub. Mus., Quebec. Female not located in either collection.

Atractodes scapiphorus. Type--Yellow label 307. 2nd Coll. Pub. Mus., Quebec. Badly broken; only thorax, coxæ in part and wings, except right fore wing, remaining.

Aulacus bilobatus. Type.-Male, yellow label 82. 2nd Coll. Pub. Mus., Quebec. Left antenna gone.

(To be continued.) 
LECTOTYPES OF THE SPECIES OF HYMENOPTERA (EXCEPT APOIDEA) DESCRIBED BY ABBÉ PROVANCHER.

BY A. B. GAHAN AND S. A. ROHWER, BUREAU OF ENTOMOLOGY, WASHINGTON, D.C.

(Continued from page 308.)

Bæoneura arietina. Type--Yellow label 1380. 2nd Coll. Pub. Mus., Quebec. Badly glued.

Banchus caudatus. Type--Female, yellow läbel 1298. 2nd Coll. Pub. Mus., Quebec.

Banchus ferrugineus. Type--Yéllow label 385. 1st Coll. Pub. Mus., Quebec.

Banchus flavovariegatus. Type-Female, yellow label 311. 2nd Coll. Pub. Mus., Quebec. Female, yellow label 379. 1st Coll. 2 other specimens.

Banchus formidabilis. Type--Yellow label 378. 2nd. Coll. Pub. Mus., Quebec. Antennæ at apex, median tarsi at apex, right hind tarsi entirely, gone.

Banchus inermis. Type-Female, yellow label 313. 2nd Coll. Pub. Mus., Quebec. 3 specimens in 1st Coll.

Banchus insignus. Type--Male, yellow label 387. 1st Coll. Pub. Mus., Quebec. Right anterior tarsus, left median tarsus at 2 nd joint, left hind tarsus and right at metatarsus, right median leg, broken off.

Banchus pallescens. Type--Male, yellow label 386. 1st Coll. Pub. Mus., Quebec. Left antenna at 5th joint, left fore leg and right hind leg gone.

Banchus polychromus. Type.-Female, yellow label 1551. 2nd Coll. Pub. Mus., Quebec.

Basalys ruficornis. Type--Yellow label 913.' 2nd Coll. Pub. Mus., Quebec.

Bassus aciculatus. Type--Female, yellow label 1565. 2nd Coll. Pub. Mus., Quebec. Lacks most of antennæ.

Bassus albicornis. Type.-Not in Pub. Mus., Quebec. unless under name, B. orbitalis Cress.

Bassus amœnus. Type.-Not in Pub. Mus., Quebec, unless under name, $B$. orbitalis Cress. 
Bassus areolatus. Type.-Not in Pub. Mus., Quebec, unless under name, Lampronota punctulata Cress.

Bassus auriculatus. Type.-Not located.

Bassus belangeri. Type.-Female, yellow label 709. 2nd Coll. Pub. Mus., Quebec.

Bassus bouleti. Type.-Not in Pub. Mus., Quebec, unless under name, Erronemus pedialis Cress.

Bassus cingulatus. Type.-Female, yellow label 985. 2nd Coll. Pub. Mus., Quebec.

Bassus costalis. Type--Female, yellow label 453. 2nd Coll. Pub. Mus., Quebec., Some legs gone.

Bassus cylindricus. Type.-Male, yellow label 103(s) and 1246 (Prov.) 2nd Coll. Pub. Mus., Quebec. Lacks left antenna.

Bassus dorsalis. Type.-Female, blue label 195(s); yellow label 1247. 2nd Coll. Pub. Mus., Quebec. Apices of antennæ gone.

Bassus elongatus. Type.-Male, yellow label 694. 2nd Coll. Pub. Mus., Quebec.

Bassus fuscitarsus. Type--Male, yellow label 349. 2nd Coll. Pub. Mus., Quebec.

Bassus humeralis. Type.-? yellow label 448. 1st Coll. Pub. Mus., Quebec. Abdomen wanting. Sex not determined. One male, yellow label 348, same species, in 2nd Coll. Species described from one female.

Bassus ichneumonoides. Type--Species badly confused, impossible to choose lectotype.

Bassus longicornis. Type.-Yellow label 932. 2nd Coll. Pub. Mus., Quebec.

Bassus mellipes. Type.-Female, yellow label 1656. 2nd Coll. Pub. Mus., Quebec. Badly mounted, antennæ gone.

Bassus pallipennis. Type-Female, yellow label 1021. 2nd Coll. Pub. Mus., Quebec.

Bassus pectoralis. Type--Yellow label 449. 1s coll. Pub. Mus., Quebec. Lacks abdomen and antennæ.

Bassus pulchripes. Type--Male, yellow label 446. 1st Coll. Pub. Mus., Quebec. Lacks apex of right antenna.

Bassus saginatus. Type.-Female, yellow label 533. 2nd Coll. Pub. Mus., Quebec. 
Bassus scapulatus. Type.-Female, yellow label 994. 2nd Coll. Pub. Mus., Quebec.

Blacus cuneatus. Type.-Female, yellow label 1592. 2nd Coll. Pub. Mus., Quebec. Antennæ broken at tip.

Blacus defectuosus. Type.-Female, yellow label 1287, blue label 734. 2nd Coll. Pub. Mus., Quebec. Anterinæ broken about 10 th joint.

Blacus longicaudus. Type.-Yellow label 1282, blue label 710. 2nd Coll. Pub. Mus., Quebec. Head and fore legs missing.

Blennocampa paupera. See Selandria.

Blepharipus cinctipes. Type--Male, yellow label 957. 2nd Coll. Pub. Mus., Quebec.

Blepharipus nigricornis. Type--Male, yellów label 1448 . 2nd Coll. Pub. Mus., Quebèc.

Brachistes crassigaster.-See Calyptus.

Brachistes submucronatus.- See Calyptus.

Bracon æqualis. Type.-Female, yellow label 551. 2nd Coll. Pub. Mus., Quebec., Apices of antennæ gone.

Bracon angelesius. Type.-Female, blue label K(s), white label 25(s), yellow label 1486. Head and wings except left hind wing, gone.

Bracon apicatus. Type.-Femalé, yellow label 554, 2nd Coll: Pub. Mus., Quebec. Lacks ovipositor, apices of antennæ and some tarsi.

Bracon auripes. Type.-Female, blue label 670 , yellow label 1571. 2nd Coll. Pub. Mus., Quebec. Right wings and head gone.

Bracon inquisitor. Type.-Female, yellow label 536. 2nd Coll. Pub. Mus., Quebec. Lacks flagella and right wings.

Bracon lævis. Type.-Female, yellow label 537. 2nd Coll. Pub. Mus., Quebec. Lacks flagella and left wings.

Bracon longicaudis. Type.-Yellow label 602. 2nd Coll. Pub. Mus., Quebec. Two specimens on same pin, top one female lacks antennæ, other one- probably male, lacks abdomen and part of antennæ.

Bracon lutus. Type.-Yellow label 552. 2nd Coll. Pub. Mus., Quebec. 
Bracon nanus. Type.-Female, yellow label 725. 2nd Coll. Pub. Mus., Quebec.

Bracon nigripectus. Type--Female, yellow label 553. 2nd Coll. Pub. Mus., Quebec. Apices of antennæ gone.

Bracon nigripes. Type.-Female, yellow label 1261. 2nd Coll. Pub. Mus., Quebec.

Bracon nitidus. Type--Male not in Coll. Female, allotype, yellow label 1026. 2nd Coll. Pub. Mus., Quebec. Female, paratype, yellow label 104. 2nd Coll. Pub. Mus., Quebec.

Bracon obliquus. Type.-Female, yellow label 541. 2nd Coll. Pub. Mus., Quebec. Dirty.

Bracon ornatus. - See Iphiaulax.

Bracon pilosipes.-Type.-Male, yellow label 1655, (also round, faded orange disk). 2nd Coll. Pub. Mus., Quebec.

Bracon politus. Type-Cat. No. 1969, U. S. N. M.

Bracon pygmæus. Type.-Female, mica tag, yellow label 555. 2nd Coll. Pub. Mus., Quebec.

Bracon rufovariegatus. Type-Male, yellow label 605 . 2nd Coll. Pub. Mus., Quebec. Female allotype without label.

Bracon sanguineus. Type-Cat. No. 1968, U. S. N. M.

Bracon striatus. Type.-Male, yellow label 724. 2nd Coll. Pub. Mus., Quebec.

Callimome fagopirum. Type--Yellow label 917. 2nd Coll. Pub. Mus., Quebec. Fair.

Callimome longicauda. Type--Yellow label 1019. 2nd Coll. Pub. Mus., Quebec. Fair.

Calyptus crassigaster. Type--Female, yellow label 1300. 2nd Coll. Pub. Mus., Quebec. Dirty.

Calyptus submucronatus. Allotype.-Female, yellow label 569. 2nd Coll. Pub. Mus., Quebec. Antennæ missing. Type male not located.

Campoplex carinatus. Type.-Female, yellow label 294. 2nd Coll. Pub. Mus., Quebec.

Campoplex flavipennis. Type.-Not in Pub. Mus., Quebec, unless under Opheletes glaucopterus Linn. Three specimens 2nd Coll., 2 in 1st Coll.

Campoplex lucens.-See Mesoleptus.

Campoplex luctuosus. Type.-Female, yellow label 335 . 1st Coll. Pub. Mus., Quebec. Head and left fore leg entirely gone. 
Campoplex marginatus.-See Limnerium.

Campoplex minor. Type.-Female, yellow label 293. 2nd Coll Pub. Mus., Quebec. Antennæ gone.

Campoplex niger. Type.-Female, yellow label 1220. 2nd Coll. Pub. Mus., Quebec. Badly broken.

Campoplex nigripes. Type.-Not in Pub. Mus., Quebec, unless under C. laticinctus Cress. Female, 2nd Coll., female 1st Coll.

Campoplex politus.-See Exolytus.

Campoplex scalarius. Type.-Female, yellow label 1219. 2nd Coll. Pub. Mus., Quebec.

Campoplex semirufus. Type.-Female, yellow label 1024 . 2nd Coll. Pub. Mus. Quebec. Antennæ, one at scape, the left at 3rd joint, middle tarsi at 2nd joint, right hind tarsus and last joint of left hind tarsus gone; abdomen broken off, stuck on pin below specimen. Allotype not located.

Campoplex unicolor.-See Mesoleptus uniformis Prov.

Campoplex vicinus. Type-Female, yellow label 291. 2nd Coll. Pub. Mus., Quebec.

Camptotera clavata. Type.-Not at present in Coll. Sent to Girault; June, 1911.

Capitonius rubriceps. Type.-Female, blue label 721(s), yellow label 1279. 2nd Coll. Pub. Mus., Quebec.

Capitonius rugosus. Type--Female Cat. No. 21433 U.S. N.M. Lacks fore wings and antennæ. Male, allotype, blue label 606, yellow label 1255. 2nd Coll. Pub. Mus., Quebec. Lacks apex of left antenna.

Centeterus tuberculifrons. Type.-Female, yellow label 249. 1st Coll. Pub. Mus., Quebec. Some verdigris.

Cephus bicinctus.-See Phyllocus.

Cephus interruptus. Type--Female, yellow label 1542. 2nd Coll. Pub. Mus., Quebec. Head gone.

Ceratosoma rufus. Type.-Female, yellow label 377. 1st Coll. Pub. Mus., Quebec. Antennæ broken at apex.

Cerceris æqualis. Type-Cat. No. 1974 U. S. N. M.

Ceropales minima. Type.-Male, blue label 124(s), yellow label 1420. 2nd Coll. Pub. Mus., Quebec. 
Ceropales superba. Type.-Harrington Coll. Paratype, yellow label 766. 2nd Coll. Pub. Mus., Quebec.

Ceroptres dorsalis. Type.-White label 56; white label 71(s); yellow label 1595. 2nd Coll. Pub. Mus., Quebec.

Charitopus facialis. Type.-Harrington Coll.

Charops fuscipennis. Type--Female, Cat. No. 1967 U. S. Nat. Mus. Right antenna broken; right hind tarsi gone; right wings glued on label.

Chelonus argentifrons. Type.-Male, yellow label 1304 . 2nd Coll. Pub. Mus., Quebec. Apices of antennæ gone.

Chelonus basicinctus. Type--Female, yellow label 906. 2nd Coll. Pub. Mus., Quebec. Left flagellum and apex of right gone.

Chelonus carinatus. Type-Female, yellow label 907. 2nd Coll. Pub. Mus. Quebec., Apex of left antennả gone. Specimen rather dirty.

Chelonus fissus. Type.-Male, yellow label 598. 2nd Coll. Pub. Mus., Quebec.

Chelonus nanus. Type.-Male, yellow label 908. 2nd Coll. Pub. Mus., Quebec.

Chelonus rufiscapus. Type--Female, yellow label 1303. 2nd Coll. Pub. Mus., Quebec.

Chiloneurus maculatipennis. Type--Harrington Coll.

Chorinæus pulchripes. Type.-Female, yellow label 1025. 2nd Coll. Pub. Mus., Quebec.

Chrysis aurichalcea. Type.-Not located.

Chrysocharis viridis. Type--Harrington Coll. Fair.

Cinctus nasutus. Type.-Blue label 769; yellow label 1328 . 2nd Coll. Pub. Mus., Quebec.

Gleonymus superbus. Type--Yellow label 1601. 2nd Coll. Pub. Mus., Quebec. Fair.

Cleptes americana. (preoc.) = provancheri Aaron. Type. - Not in Quebec. May be in Philadelphia.

Clistopyga canadensis. Type.-Female, yellow label 396. 2nd Coll. Pub. Mus., Quebec.

Clistopyga truncata. Type.-Female, yellow label 1001. 2nd Coll. Pub. Mus., Quebec.

(To be continued.) 
broader and more approximate on the sternal side pieces. Legs rather slender, femora not toothed, terminal joint of tarsi projecting a distance rather less than the length of the lobes of the preceding joint; claws simple.

Length $1.8 \mathrm{~mm}$.; width $.9 \mathrm{~mm}$.

Aweme, Manitoba, Sept. 8, (Criddle).

A small species belonging to the squamulatus group of Dietz, in which it may best follow persimilis, though not agreeing closely with any species of the group. The small size and lack of true scales on the upper surface distinguishes it from all but albopilosulus, which differs in its erect, coarser vestiture among other characters.

LECTOTYPES OF THE SPECIES OF HYMENOPTERA (EXCEPT APOIDEA) DESCRIBED BY ABBÉ PROVANCHER.

BY A. B. GAHAN AND S. A. ROHWER, BUREAU OF ENTOMOLOGY, WASHINGTON, D.C.

(Continued from page 336 .)

Coccophagus brunneus. Type--Yellow label 1384. 2nd Coll. Pub. Mus., Quebec. Badly glued.

Coccophagus compressicornis. Type-Harrington Coll.

Coccophagus pallipes. Type--Yellow label 1389; blue 783(s). 2nd Coll. Pub. Mus., Quebec. Head gone.

Coleocentrus mellipes. Type-- Not located.

Coleocentrus quebecensis. Type.-Not in Pub. Mus., Quebec., unless under $C$. pettiti Cress.

Coleocentrus rufus. Type--Female, yellow label 456. 1st Coll. Pub. Mus., Quebec.

Copelus paradoxus.-See Helorus.

Copidosoma pallipes. Type--Harrington Coll.

Crabro aciculatus. Type.-Female, yellow label 813. 2nd Coll. Pub. Mus., Quebec. Male, allotype, without label.

Crabro niger. Type.-Female, blue-green label 852(s), yellow label 1660. 2nd Coll. Pub. Mus., Quebec.

Crabro 4-maculatus.-See C. 4-punctatus. Type the same specimen.

November, 1917 
Crabro 4-punctatus. Type-Female, yellow label 807. 2nd Coll. Pub. Mus., Quebec. See C. 4-maculata. See Fauna p. 654 and table 653 , both names used.

Cratospila aciculata. Type.-No specimen. Pin with name label. 2nd Coll. Pub. Mus., Quebec. Probably returned to collector.

Cratospila brevicauda. Type--Female, yellow label 1273. 2nd Coll. Pub. Mus., Quebec. Lacks apex of left flagellum.

Cratospila caudata. Type.-Female, yellow label 606. 2nd Coll. Pub. Mus., Quebec. Lacks flagella.

Cremastus fusiformis. Type.-Female, yellow label 306. 2nd Coll. Pub. Mus., Quebec. Antennæ broken at apex.

Cremastus longicaudus. Type. - Female, yellow label 1050. 2nd Coll. Pub. Mus., Quebec.

Cremastus mellipes. Type.-Female, yellow label 363. 1st Coll. Pub. Mus., Quebec. Antennæ broken near middle; fore wings gone; badly pinned. Female. 2nd Coll. antennæ broken.

Cremastus rectus. Type-Yellow label 361. 1st Coll. Pub. Mus., Quebec. Left antenna at scape, left hind leg at coxa and abdomen gone.

Cremastus royi. Type-Female, yellow label 674. 2nd Coll. Pub. Mus., Quebec.

Cryptus affabilis. Type--Female, yellow label 258. 2nd Coll. Pub. Mus., Quebec.

Cryptus albonotatus. Type--Not located.

Cryptus amblytelarius. Type-Female, yellow label 1204. 2nd Coll. Pub. Mus., Quebec.

Cryptus annulatus. Type--Female, yellow label 291. 1st Coll. Pub. Mus.; Quebec. Lacks right fore wing.

Cryptus apicatus. Type.-Female, yellow label 246. 2nd Coll. Pub. Mus., Quebec. Lacks right antenna.

Cryptus belangeri. Type.-Not in Pub. Mus., Quebec, unless under name $C$. nuncius Say. One specimen.

Cryptus brevicornis. Nat. Can., Vol. 7, p. 176.--See Phygadeuon impressus.

Cryptus brevicornis. Of index p. 432. Type.-Female, old rcse label 79; yellow label 1202. 2nd Coll. Pub. Mus., Quebec. 
Cryptus canadensis. Type.-Male, yellow label 248. 2nd Coll. Pub. Mus., Quebec. Lacks left antenna.

Cryptus certus. Type.-Not in Pub. Mus., Quebec, unless under name $C$. fungor Nort. Two damaged specimens.

Cryptus cinctus. Type--Male, yellow label 292. 1st Coll. Pub. Mus., Quebec. Lacks apex of right antenna.

Cryptus circumcinctus. Type--Male, yellow label 516. 2nd Coll. Pub. Mus., Quebec. Lacks antennæ:

Cryptus collaris. Type--Male, blue label 121, yellow label 1206. 2nd Coll. Pub. Mus., Quebec.

Cryptus dubius. Type.-Not in Pub. Mus., Quebec. Probably returned to collector. (Geddes).

Cryptus eburneifrons. Type.-Male, yellow label 517. 2nd Coll. Pub. Mus., Quebec. Lacks most of antennæ.

Cryptus elongatus. Type.-Male, yellow label 670. 2nd Coll. Pub. Mus., Quebec. Lacks apices of antennæ and hind legs.

Gryptus erythropygus. Type.-Not in Pub. Mus., Quebec. Probably in Harrington Coll.

Cryptus exilis. Type--Male, yellow label 283. 1st Coll. Pub. Mus., Quebec.

Cryptus flavipectus. Type.-Not in Pub. Mus., Quebec, unless under Ichneumon scitulus Cress.

Cryptus fletcheri. Type.-Female, white label 115; also "Cryptus o fletcheri Prov. Type.-Victoria, V. I." in hand other than Provancher's. 2nd Coll. Pub, Mus., Quebec.

Cryptus gracilis. Type.-Not in Pub. Mus., Quebec. Probably in Harrington Coll.

Cryptus ignotus. Type--Male, Harrington Coll. Pink label "P 423." Part of left antenna gone.

Cryptus imitator. Type.-Female, yellow label 296. 1st Coll. Pub. Mus., Quebec.

Cryptus incognitus. Type--Blue label 39 ; yellow label 1205. 2nd Coll. Pub. Mus., Quebec. Lacks left flagellum.

Cryptus insignis. Type.-Not in Pub. Mus., Quebec, unless under Phygadeuon blakei Cress.

Cryptus latus. Type.-Not in Pub. Mus., Quehec, unless under Phygadeuon occidentalis. 
Cryptus linearis. Type.-Male, old rose label 49. Yellow label 1207. 2nd Coll. Pub. Mus., Quebec. Part of antennæ gone.

Cryptus longicaudus. Type.-Female, Harrington Coll. Pink label "P. 375."

Cryptus mellicoxus. Type--Male, yellow label 1209. 2nd Coll. Pub. Mus., Quebec. Apices of antennæ gone; abdomen glued on number label.

Gryptus mellipes. Type.-Female, Harrington Coll. Pink làbel "P. 392." Abdomen wanting, wings except left hind wanting.

Cryptus montivagus. Type.-Female, yellow label 251. 2nd Coll. Pub. Mus., Quebec. Lacks most of flagella.

Cryptus mundus. Type.-Male, yellow label 245. 2nd Coll. Pub. Mus., Quebec. Lacks part of antennæ and right hind leg.

Cryptus nigricornis. Type.-Male, yellow label 294. 1st Coll. Pub. Mus., Quebec. Lacks left antenna beyond 4th joint.

Cryptus nigricoxus. Type-Male, yellow label 1553. 2nd Coll. Pub. Mus., Quebec.

Cryptus notatus. Type--Male, yellow label 254. 2nd Coll. Pub. Mus., Quebec.

Cryptus occidentalis. - See Phygadeuon occidentalis.

Cryptus ornatus. Type.-Not in Pub. Mus., Quebec.

Cryptus osculatus. Type.-Male, yellow label 281. 1st Coll. Pub. Mus., Quebec. Lacks right flagellum.

Cryptus pentagonalis. Type.-Harrington Coll. Male, pink label 425. Female, pink label 431.

Cryptus perditus. Type--Male, Harrington Coll. Pink label "P. 442." Lacks antennæ and right fore wing; other wing folded and hard to see.

Cryptus pubescens. Type.-Male, Harrington Coll. Pink label "P. 424."

Cryptus quebecensis. Type.-Not in Coll. unless under name Ichneumon velox Cress. Two females. 1st Coll. fair.

Cryptus rectus. Type.-Male, yellow label 1208. 2nd Coll. Pub. Mus., Quebec. Lacks left flagellum. 
Cryptus ruficornis. Type.-Male, yellow label 519. 2nd Coll. Pub. Mus., Quebec. Some verdigris.

Cryptus rufoannulatus. Type--Female, yellow label 286 . 1st Coll. Pub. Mus., Quebec.

Cryptus rufus. Type--Male, yellow label 259. 2nd Coll. Pub. Mus., Quebec. Lacks right flagellum.

Cryptus scutellatus. Type.-Male, yellow label 282. 1st Coll. Pub. Mus., Quebec. Lacks apices of antennæ some tarsi, and abdomen has been glued on.

Cryptus segregatus. Type--Male, Harrington Coll. Pink label "P. 422."

Cryptus sericeifrons. Type--Female, yellow label 515 . 2nd Coll. Pub. Mus., Quebec. Most of flagellum gone. No male in Coll.

Cryptus signatus.- See Phygadeuon signatus.

Cryptus sordidus. Type--Female, Harrington Coll. Pink label "P. 373." Abdomen off but glued on card.

Cryptus soriculatus. Type.-Male, yellow label 697. 2nd Coll. Pub. Mus., Quebec. Lacks right antenna.

Cryptus spissicornis. (Addit. 1886, p. 68, not suppl. p. 361). Type.-Not in Pub. Mus., Quebec, unless under Cryptus crassicornis of which there is one specimen agreeing with description of spissicornis.

Cryptus spissicornis. (1888, suppl. p. 361 not Addit., 1886, p. 68). Type.-Female, yellow label 1582. 2nd Coll. Pub. Mus., Quebec.

Cryptus 3-annulatus. Type.-Not in Pub. Mus., Quebec. Probably in Harrington Coll.

Cryptus varius. Type.-Not in Pub. Mus., Quebec, unless under name Cryptus atricollaris Walsh.

Cteniscus apicatus. Type--Male, yellow label 341. 2nd Coll. Pub. Mus., Quebec. Lacks antennæ.

Cteniscus concolor. Type--Female, yellow label 321 . 2nd Coll. Pub. Mus., Quebec. Under name Mesoleptus concolor Cress. Lacks most of antennæ.

Cteniscus crassipes. Type.-Not in Pub. Mus., Quebec. Probably returned to collector. 
Cteniscus rufus. Type.:-Public Mus., Quebec. Data from pin not obtained.

Ctenopelma sanguinea. Type--Pub. Mus., Quebec. Data from pin not obtained.

Cylloceria lemoinei. Type.-Male, yellow label 470. 2nd Coll. Pub. Mus., Quebec. Lacks apices of antennæ.

Cyrtocentrus quebecensis. Type.-Female, yellow label 693. 2nd Coll. Pub. Mus., Quebec. Antennæ wanting beyond third joint.

Dacnusa crassitela. Type.-Female, yellow label 1299. 2nd Coll. Pub. Mus., Quebec. Antennæ broken, right at scape, left at seventh joint.

Dacnusa spatulata.' Type.-Female, yellow label 1305, blue label 669. 2nd Coll. Pub. Mus., Quebec. Antennæ broken at tips.

Decatoma basilaris. Type.-Not in Pub. Mus., Quebec, unless under Isosoma hordei.

Diastrophus piceus. Type.-White label 3(s); yellow label 1325. 2nd Coll. Pub. Mus., Quebec.

Diastrophus 5-costatus. Type.-Not located.

Dimicrostrophis nigricornis. Type--Mica point, blue label 759(s); yellow label 1664. 2nd Coll. Pub. Mus., Quebec.

Dineura americana. Type.-Yellow label 639. 2nd Coll. Pub. Mus., Quebec. Lacks left flagellum.

Dinocamptus linearis. Type--Yellow label 1275. 2nd Coll. Pub. Mus., Quebec. Thorax broken by pin and abdomen crushed out of shape at apex. Sex of type uncertain but believed to be, female.

Dinotus acutus. Type.-Yellow label 1385. 2nd Coll. Pub. Mus., Quebec. Badly glued; another specimen in better condition.

Dolichoderus borealis. Type--Yellow label 1603. 2nd Coll. Pub. Mus., Quebec.

Doryctes atripes. Type.-Female, yellow label 1262. 2nd Coll. Pub. Mus., Quebec. 
Doryctes bæticatus. Type--Yellow label 557. 2nd Coll. Pub. Mus., Quebec. Abdomen wanting.

Doryctes cingulatus. Type--Female, yellow label 556 . 2nd Coll. Pub. Mus., Quebec. Lacks flagella.

Doryctes fartus. Type.-Female, yellow label 558. 2nd Coll. Pub. Mus., Quebec.

Doryctes macilentus. Type--Female, yellow label 559. 2nd Coll. Pub. Mus., Quebec. No male in collection.

Doryctes pallipes. Type.-Female, yellow label 1569 . 2nd Coll. Pub. Mus., Quebec.

Echthrus canadensis. Type--Female, yellow label 468. 2nd Coll. Pub. Mus., Quebec. Some verdigris.

Echthrus luctuosus. Female, yellow label 521. 1st Coll. Pub. Mus., Quebec. Lacks apex of left antenna and abdomen.

Echthrus nigricornis. Type.-Male, yellow label 421. 2nd Coll. Pub. Mus., Quebec. Fair.

Echthrus pediculatus. Type.-Female, yellow label 937. 2nd Coll. Pub. Mus., Quebec.

Echthrus provancheri. Type--Female, yellow label 1143; printed name label. 2nd Coll. Pub. Mus., Quebec. Considered as Brodie's species. The description was first published by Provancher and there is no indication that it was quoted from Brodie. Provancher indicates that it is his description so the species should, unfortunately, be accredited to Provancher.

Echthrus rubripes. Type.-Female, yellow label 1064. 2nd Coll. Pub. Mus., Quebec. 'Left wings gone.

Eclytus pleuralis. Type.-Female, yellow label 410. 1st Coll. Pub. Mus., Quebec.

Eclytus robustus. Type.-Female, yellow label 996. 2nd Coll. Pub. Mus., Quebec. Abdomen and part of antennæ gone.

Elis dives. Type.-Female, Cat. No. 1971, U. S. Nat. Mus. Elis 4-cinctus. Type--Male, Cat. No. 1972, U. S. Nat. Mus. Emphytus hullensis. Type--Harrington Coll.

Emphytus nigristigma. Type.-Yellow label 1543; name label "Nematus nigristigma." 2nd Coll. Pub. Mus., Quebec. Provancher's catalogue proves this. 
Emphytus pallipes. Type.-Female, yellow label 35. 1st Coll. Pub. Mus., Quebec. Specimen without label, paratype.

Ephedrus completus. Type--See introduction (Aphidiinæ).

Ephedrus incompletus. Type. - See introduction (Aphidiinæ).

Ephialtes variatipes. Type.-Harrington Coll. teste Davis. Not in Pub. Mús., Quebec:

Epirhyssa clavata. Type.-Female, yellow label 1260. 2nd Coll. Pub. Mus., Quebec. Lacks abdomen and apices of antennæ.

Epirhyssa crevieri. Type.-Male, yellow label 388. 2nd Coll. Pub. Mus., Quebec.

Epyris formicoides. Type--White label "Hull 25-8-84 Rus"; blue label 312; yellow label 1028. 2nd Coll. Pub. Mus., Quebec.

Eypris prolongatus. Type--Yellow label 744(s). 2nd Coll. Pub. Mus., Quebec. Abdomen gone.

Eriocampa marginata.-See Selandria.

Eriocampa superba. Type-Female, yellow label 1544. 2nd Coll. Pub. Mus., Quebec.

Erronemus bedardi. Type--Female, yellow label 658 . 2nd Coll. Pub. Mus., Quebec.

Erronemus marginatus. Type.-Female, yellow label 989. 2nd Coll. Pub. Mus., Quebec.

Erronemus tristis. Type.-Female, blue label 594. 2nd Coll. Pub. Mus., Quebec. Left antenna gone.

Eubadizon basilare. Type:-Not located.

Eubadizon californicus. Type.-Female, yellow label 1489. 2nd Coll. Pub. Mus., Quebec. Antennæ broken beyond middle, head broken off and mounted on tag.

Eubadizon gracilis: Type.-Female, yellow label 603. 2nd Coll. Pub. Mus., Quebec. One antenna and left hind tarsi broken.

Eucerceris insignis. Type-Male, white label $77(\mathrm{~s})$; yellow label 1626. 2nd Coll. Pub. Mus., Quebec.

Eucharis gibbosa. Type.-Yellow label 623. 2nd Coll. Pub. Mus., Quebec. Head gone. 
Eucoila minor. Type--Not located.

Eulophus ramosus. Type.-Yellow label 924. 2nd Coll. Pub. Mus., Quebec. Fair.

Eulophus tricladus. Type--Harrington Coll. Paratype, yellow label 1374; blue label 754. 2nd Coll. Pub. Mus., Quebec. Fair.

Eumenes cinctus. Type--Female, round disk, yellow label 1643. 2nd Coll. Pub. Mus., Quebec. Poor condition, dirty, etc.

Eumenes crucifera. Type--Female Cat. No. 1978, U. S. Nat. Mus.

Eumenes flavopictus. Type.-Female, round disk, yellow label 1644. 2nd Coll. Pub. Mus., Quebec.

Eumenes impunctus. Type--Female, round disk, yellow label 1642. 2nd Coll. Pub. Mus., Quebec.

Euphorus cephalicus. Type.-Male, yellow label 1118. 2nd Coll. Pub. Mus., Quebec. One antenna gone, other showing 8 joints.Poorly tag-mounted.

Eup̄horus punctatus. Type--Male, yellow label 991. 2nd Coll. Pub. Mus., Quebec. Badly pinned; head missing.

Euplectrus lucens. Type--Yellow label 1369: 2nd Coll. Pub. Mus., Quebec. Fair.

Euplectrus mellipes. Type-Female, Harrington Coll. Antennæ broken, one complete to fourth funicle joint. Paratype.Yellow label 1363. 2nd Coll. Pub. Mus., Quebec. Two specimens, both badly glued.

Euplectrus viridæneus. Type--Yellow label 1382; blue 771(s). 2nd Coll. Pub. Mus., Quebec. Fair.

Eurytoma conica. Type--Harrington Coll.

Eurytoma nigricoxa. Type.-Harrington Coll.

Eustalocerus fasciatus. Type.-Female, blue label 701(s), yellow label 1281. 2nd Coll. Pub. Mus., Quebec. Lacks flagella.

Eustalocerus longicornis. Type.-Female; yellow label 1280. 2nd Coll. Pub. Mus., Quebec. Antennæ and hind tarsi, broken.

Eustalocerus petiolatus. Type. - Female, yellow label 1083. 2nd Coll. Pub. Mus., Quebec. Left antenna, median and posterior legs on left, missing. 
Eustalocerus tauricornis.' Type.-Female, yellow label 566. 2nd Coll. Pub. Mus., Quebec. One antenna gone.

Euura nigra. Type.-Yellow label 1538. 2nd Coll. Pub. Mus., Quebec. Another specimen with small, white label "161."

Euxorides vancouverensis. Type-Female, yellow label 1556. 2nd Coll. Pub. Mus., Quebec. Lacks flagellum.

Exenterus canadensis. Type- - Not located. Allotype.Female, yellow label 953. 2nd Coll. Pub. Mus., Quebec.

Exenterus hullensis. Type--Male, Harrington Coll. Pink label "P. 581." Allotype.-Female, blue label 468, yellow label 1238. 2nd Coll. Pub. Mus., Quebec.

Exetastes albitarsis. Type.-Female, yellow label 376. 1st Coll. Pub. Mus., Quebec.

Exetastes brevipennis. Type.-Not in Pub. Mus., Quebec, unless under Mesostenus promptus Cress.

Exetastes clavatus. Type- - Not located.

Exetastes matricus. Type--Female, yellow label 531. 2nd Coll. Pub. Mus., Quebec. Right antenna at scape, left before the middle, anterior and median legs on right at coxæ gone.

Exetastes rufofemoratus. Type-Female, yellow label 310. 2nd Coll. Pub. Mus., Quebec.

Exetastes rufus.- See Ceratosoma.

Exochilum nigrum. Type--Female, yellow label 279. 2nd Coll. Pub. Mus., Quebec: Left antenna gone, right broken at apex, hind tarsi broken at third joint.

Exochus rufomaculatus. Type.-Female, blue label 562 . 2nd Coll. Pub. Mus., Quebec.

Exolytus politus. Type.-Female, yellow label 365. 1st Coll. Pub. Mus., Quebec.

Exyston marginatum. Type.-Not in Pub. Mus., Quebec. Probably returned to collector.

Exyston variatus. Type.-Male, yellow label 324. 2nd Coll. Pub. Mus., Quebec. 
LECTOTYPES OF THE SPECIES OF HYMENOPTERA (EXCEPT APOIDEA) DESCRIBED BY ABBÉ PROVANCHER.

BY A. B. GAHAN AND S. A. ROHWER, BUREAU OF ENTOMOLOGY, WASHINGTON, D. C.

(Continued from page 400.)

Figites inermis. Type.-Female, yellow label 619. 2nd Coll. Pub. Mus. Quebec.

Formica pallitarsis. Type--Yellow label 925. 2nd Coll. Pub. Mus., Quebec. Fair.

Galesus quebecensis. Type--Yellow label 912. 2nd Coll. Pub. Mus., Quebec.

Glypta californica. Type--Female, white label 8, yellow label 1292. 2nd Coll. Pub. Mus., Quebec.

Glypta ruficornis. Type--Male, yellow label 490. 1st Coll. Pub. Mus., Quebec. Bears name label Glypta macra Cress.

Glypta rugulosa. Type--Yellow label 986. 2nd Coll. Pub. Mus., Quebec. Thorax and wings present.

Glypta tricincta. Type--Not located.

Gonatopus decipiens. Type--Yellow label 1332. 2nd Coll. Pub. Mus., Quebec. Badly glued.

Gorytes armatus. Type.-Male, old rose label $256(\mathrm{~s})$, blue label 845(s), yellow label 1434. 2nd Coll. Pub. Mus., Quebec.

Gorytes eximius. Type.-Male, white label 29(s), yellow label 1436. 2nd Coll. Pub. Mus., Quebec.

Gorytes laticinctus. Type.-Not located. Probably returned to collector.

Gorytes maculatus. Type--Male, yellow label 1726. 2nd Coll. Pub. Mus., Quebec.

Gorytes ruficornis. Type--Female, white label 36(s), yellow label 1435. 2nd Coll. Pub. Mus., Quebec.

Haltichella viridis. Type.-Blue label 110(s); yellow, 1394. 2nd Coll. Pub. Mus., Quebec.

Hedylus politus. Type.-See Introduction (Opiinæ).

Helorus paradoxus. Type.-Female, yellow label 971. 
2nd Coll. Pub. Mus., Quebec. Lacks head. Paratype in good condition.

Hemiteles aciculatus. Type - Male, Harrington Coll. Pink label "P. 426." Lacks right antenna and apex of left.

Hemiteles caudatus. Type.-Female, yellow label 306. 1st Coll. Pub. Mus., Quebec. Lacks abdomen.

Hemiteles crassus. Type.-Male, yellow label 720. 2nd Coll. Pub. Mus., Quebec. Some tarsi gone.

Hemiteles debilis. Type--Harrington Coll. One antennæ gone, other broken at apex.

Hemiteles declivus. Type. - Female, Harrington Coll. Antennæ gone. Paratype.-Old rose label 33, yellow label 1199. 2nd Coll. Pub. Mus., Quebec.

Hemiteles depressus. Type--Not located.

Hemiteles gigas. Type--Male, Harrington Coll. Pink label "P. 411."

Hemiteles humeralis. Type-Female, yellow label 233. 2nd 'Coll. Pub. Mus., Quebec.

Hemiteles longicornis. Type-Female, yellow label 671 . 2nd Coll. Pub. Mus., Quebec.

Hemiteles mandibularis. Type.-Female, yellow label 307. 1st Coll. Pub. Mus., Quebec.

Hemiteles mucronatus. Type--Male, blue label 213, yellow label 1198. 2nd Coll. Pub. Mus., Quebec. Lacks left fore wing.

Hemiteles nigricans. Type--Male, yellow label 673. 2nd Coll. Pub. Mus., Quebec. Apices of antennæ gone, wings' crumpled.

Hemiteles orbicularis. Type.-Not in Pub. Mus., Quebec, unless under Stilpnus americanus Prov.

Hemiteles ovalis. Type.-Female, yellow label 316. 1st Coll. Pub. Mus, Quebec. Lacks right and apex of left antenna. Allotype.-Not located.

Hemiteles pallipennis. Type--Female, yellow label 672 . 2nd Coll. Pub. Mus., Quebec. Lacks left antenna and apex of right. Allotype--In good condition, without labels. 2nd Coll. Pub. Mus., Quebec.

Hemiteles parvus. Type-Male, yellow label 226. 2nd Coll. Pub. Mus., Quebec. Fair. 
Hemiteles rüficoxus. Type--Female, yellow label 228 . 2nd Coll. Pub. Mus., Quebec. Some verdigris.

Hemiteles scabrosus. Type--Female, yellow label 225. 2nd Coll. Pub. Mus., Quebec. Lacks apex of right flagellum.

Hemiteles semifurus. Type.-Female, yellow label 314 . 1st Coll. Pub. Mus., Quebec. Without head.

Hemiteles sessilis. Type -Female, yellow label 309. 1st Coll. Pub .Mus., Quebec.

Hemiteles subspinosus. Type--Female, Yellow label 232. 2nd Coll. Pub. Mus, Quebec. Lacks antennæ.

Hemiteles tener. Type.-Male, yellow label 311. 1st Coll. Pub. Mus., Quebec. Somewhat damaged.

Hemiteles utilis. Type.-See H. depressus Prov.

Herpestomus pyriformis. Type.-Female, yellow label 200. 2nd Coll. Pub. Mus., Quebec.

Heteropelma longipes.Type.-Cat. No. 1966, U. S. Nat. Mus.

Holcopelte albipes. Type.-Yellow label 1378; blue 774(s). 2nd Coll. Pub. Mus., Quebec. Fair.

Hoplismenus impar. Type:-Pub. Mus., Quebec. Data not obtained.

Hoplismenus scutellatus. See Ichneumon.

Hoplismenus stygicus. Type.-Not located, probably returned to collector.

Hoplisus angustus. Type--Male, yellow label 1688. 2nd Coll. Pub. Mus., Quebec.

Hoplocampa canadensis. See Selandria.

Ichneumon absconditus. Type.-Male, yellow label 1210 . Mus. Pub. Instruction, Quebec. Right antenna broken, left fore wing missing, thorax crushed but complete, hind tarsi broken, 3 basal joints of right present.

Ichneumon adjunctus. Type.-Female, blue label 93, yellow label 1189? (or one hundred and eighty-nine). 2nd Coll. Pub. Mus., Quebec.

Ichneumon æqualis. Type.-Not in Pub. Mus., Quebec, unless under Amblyteles nubivagus Cress.

Ichneumon annulatus. Type.-Male, yellow label 211. 1st Coll. Pub. Mus., Quebec. Right antenna broken off at scape. 
Ichneumon approximans. Type.-Male, old rose label 31 , yellow label 1193. 2nd Coll. Pub. Mus., Quebec.

Ichnuemon aterrimus. Type.-Not in Quebec or Ottawa. Probably returned to collector.

Ichneumon bimaculatus. Type- -Male, blue label 380 , yellow label 1580. 2nd Coll. Pub. Mus., Quebec. Right antenna, front legs, left middle leg and left hind wing missing.

Ichneumon bimembris. Type.-Female, yellow label 99 . Pub. Mus., Quebec.

Ichneumon calcaratus. Type.-Not in Pub. Mus., Quebec, unless under Hoplismenus morulus Say.

Ichneumon caudatus. Type-Female, yellow label 139. 2nd Coll. Pub. Mus., Quebec. Verdigris.

Ichneumon cervulus. Type--Male, yellow label 134 . 2nd Coll. Pub. Mus., Quebec.

Ichneumon cinctipes. Type.-Not in Pub. Mus., Quebec, unless under $I$. navus. One specimen female, yellow label 161. 1st Coll. Pub., Mus., Quebec.

Ichneumon cinctitarsis. Type--Male, yellow label 112 . Pub. Mus., Quebec: Right antenna missing.

Ichneumon citatus. Type--Male, yellow label 162. 1st Coll. Pub. Mus., Quebec. Lacks right antenna.

Ichneumon citrinus. Type--Female, Harrington Coll. Antennæ and wings on one side gone, abdomen broken off, mounted on label.

Ichneumon clapini. Type--Not in Pub. Mus., Quebec, unless under Ichneumon milvus.

Ichneumon cressoni. Type.-Not in Pub. Mus., "Quebec, unless under $I$. velox Cress.

Ichneumon decoratus. Type-Male, yellow label 135. 2nd Coll. Pub. Mus., Quebec. Right antenna broken.

Ichneumon erythropygus. Type.-Not in Pub. Mus., Quebec, unless under Platylabus thoracicus Cress.

Ichneumon fortis. Type.-Not in Pub. Mus., Quebec, unless under Ichneumon centrator Say.

Incheumon hæsitans. Type.-Not in Pub. Mus., Quebec, unless under Ichneumon funestus Cress. 
Ichneumon humilis. Type.-Female, yellow label 138. 2nd Coll. Pub. Mus., Quebec. Antennae gone.

Ichneumon lachrymans. Type--Male, yellow label 136 . 2nd Coll. Pub. Mus., Quebec.

Ichneumon lividulus. Type-Female, yellow label 144 . 2nd Coll. Pub. Mus., Quebec.

Ichneumon lobatus. Type--Not in Pub. Mus., Quebec, unless under. Ichneumon duplicatus Say.

Ichneumon magdalensis. Type--Not located.

Ichneumon marianapolitanensis. Type.-Not in Pub. Mus., Quebec, unless under Amblyteles rufizonatus Cress.

Ichneumon mellicoxus. Type. - Not in Pub. Mus., Quebec, unless under Ichneumon puerilis Cress.

Ichneumon mucronatus. Type--Male, yellow label 214. 1st Coll. Pub. Mus., Quebec. Antennæ, left front wing, hind legs, median tarsus on right leg, left anterior tibiæ and tarsi missing.

Ichneumon nigripes. Type--Male, yellow label 987. 2nd Coll. Pub. Mus., Quebec. Antennæ broken near middle.

Ichneumon nigrovariegatus. Type-Female yellow label 137. 2nd Col'. Pub. Mus., Quebec. Right antenna except scape gone, left antenna broken off near middle, abdomen glued on.

Ichneumon nitidus. Type.-Not in Pub. Mus., Quebec, unless under Amblyteles electus Cress.

Ichneumon ontariensis. Type--Male, yellow label 1191, pink label 30. Pub. Mus., Quebec.

Ichneumon paradoxus. Type.-Not located.

Ichneumon pilosulus. Type--Female, yellow label 168. 1st Coll. Pub. Mus., Quebec. Antennæ broken (one at 4th joint and one at middle) and left hind leg gone.

Ichneumon placidus. Type.-Male, yellow label 142. 2nd Coll. Pub. Mus., Quebec.

Ichneumon pomilius. Type--Male, yellow label 123. 2nd Coll. Pub. Mus., Quebec.

Ichneumon proximus. Type-Female, yellow label 163. 2nd Coll. Pub. Mus., Quebec.

Ichneumon quadripunctatus. Type- - Not located. Probably overlooked in Harrington Coll.

Ichneumon saguenayensis. Type--Not located. 
Ichneumon scutellatus. Type.-Male, yellow label 167 . 2nd Coll. Pub. Mus., Quebec. Right antenna and right hind tarsus gone.

Ichneumon similaris. Type.-Male, yellow label 113. 2nd Coll. Pub. Mus., Quebec.

Ichneumon stygicus. Type--Yellow label 167. 1st Coll. Pub. Mus., Quebec.

Ichneumon trizonatus. Type-Male, yellow label 118 2nd Coll. Pub. Mus., Quebec.

Ichneumon ustus. Allotype--Male, yellow label 510. 2nd Coll. Pub. Mus., Quebec.

Ichneumon vagans. Type--Yellow label 170. 1st Coll. Pub. Mus., Quebec. Apex of right antenna gone.

Ichneumon vancouverensis. Type-Ent. Branch, Dept. Agr., Ottawa.

Ichneumon varipes. Type.-Not in Pub. Mus., Quebec, unless under Ichneumon w-album Cress.

Ichneumon vescus. Type.-Male, yellow label 122. 2nd Coll. Pub. Mus., Quebec.

Iphiaulax americanus. Type.-Female, yellow label 1566 . 2nd Coll. Pub. Mus., Quebec. Left fore wing loose.

Iphiaulax ornatus. Type.-Female, yellow label 542. 2nd Coll. Pub. Mus., Quebec.

Ischnus impressus. See Phygadeuon.

Ischnus lentus. Type.-Not in Pub. Mus. of Quebec unless under Cryptus limatus Cress.

Ischnus parvus. See Hemiteles.

Ischnus placidus. Type.-Not in Coll. Pub. Mus., Quebec, unless under Phygadeuon rectus Prov.

Ischnus pyriformis. See Herpestomus.

Ischnus ruficornis. See Phygadeuon.

Ischnus scutellatus. See Platylabus.

Ischnus variegatus. Type.-Not in Pub. Mus., Quebec, unless under Ichneumon w-album Cress.

Isostasius canadensis. Type.-Not located.

Joppa canadensis. Type.-Not in Pub. Mus., Quebec, unless under Ichneumon insolens Cress. 
Labidia columbiana. Type.-Pin with yellow label 1152. 2nd Coll. Pub. Mus., Quebec. Specimen has been destroyed.

Lampronota albifacies. Type.-Yellow label 409. Name label Lampronota pleuralis. Cress. 2nd Coll. Pub. Mus., Quebec. Proved by Prov. catalogue.

Lampronota humeralis. Type.-Male, yellow label 417. 2nd Coll. Pub. Mus., Quebec. Lacks antennæ.

Lampronota marginata. Type.-Female, yellow label 954. 2nd Coll. Pub. Mus., Quebec.

Lampronota nigricornis. Type.-Female, yellow label 501. 1st Coll. Pub. Mus., Quebec. Lacks apical half of right antenna.

Lampronota nigripes. Type.-Not in Pub. Mus., Quebec. Probably returned to collector.

Lampronota rufipes. Type.-Provancher did not describe this as a new species. Two females, Pub. Mus., Quebec. One, yellow label 503, 1st Coll.; other, yellow label 532, 2nd Coll. Both under name label Lampronota rufipes Cress.

Larra minor. Type.-Male, blue label 77 (s), yellow label 1430. 2nd Coll. Pub. Mus., Quebec.

Larra quebecensis. Type.-Not located.

Larra rufipes. Type-Female, white label 99(s); yellow label 1715. 2nd Coll. Pub. Mus., Quebec.

Leptobates canadensis. Type.-Not in Pub. Mus., Quebec, unless under Phygadeuon signatus Prov.

Leptothorax canadensis. Type.-Not located.

Limneria argentea. Type.-Female, yellow label 471. 2nd Coll. Pub. Mus., Quebec. Antennæ broken at tip, median, and hind legs on left gone at coxæ.

Limneria basilaris. Type.-Male, yellow label 449. - 2nd Coll. Pub. Mus., Quebec. Right antenna and left median leg missing.

Limneria brevicauda. Type.-Female, blue label 252 (apparently, blotted), yellow label 1221. 2nd Coll. Pub. Mus., Quebec. Left antenna gone at scape.

Limneria clavata. Type--Female, yellow label 301. 2nd Coll. Pub. Mus., Quebec. Lacks left antenna at scape, right at apex, anterior and median legs on left.

(To be continued) 
fifth with a more diffuse band; apex with a pair of entirely separate parallel red spines, curved downward; venter red, black apically. (Bruner, 17).

The basal nervure meets the transversomedian; the marginal cell is obliquely truncate and appendiculate, exactly as in Pasites. The hind legs are much more slender than in Pasites maculatus, and the outer apical angle of hind tibia is much more salient. The face, though broad, is not so broad as in the Pasites.

\section{LECTOTYPES OF HYMENOPTERA (EXCEPT APOIDEA) DESCRIBED BY ABBE PROVANCHER.}

BY A. B. GAHAN AND S. A. ROHWER, WASHINGTON, D.C.

(Continued from vol. XLIX, page 433.)

Limneria compacta. Type.-Female, Ent. Branch, Dept. Agr., Ottawa.

Limneria crassicornis. Type--Male, yellow label 1222. 2nd Coll. Pub. Mus., Quebec. Left antenna beyond middle, right anterior leg at trochanter, left median tarsus and hind tarsi broken off.

Limneria dentata. Type.-Female, yellow label 451. 2nd Coll. Pub. Mus., Quebec. Right antenna gone at scape.

Limneria distincta. Type--Female, yellow label 1060. 2nd Coll. Pub. Mus., Quebec. Right antenna at scape, left at 6 th joint, gone.

Limneria excavata. Type.-Not in Pub. Mus., Quebec, unless under Limneria valida Cress.

Limneria flavipes. Type.-Male, yellow label 358. 1st Coll. Pub. Mus., Quebec. Right antenna beyond middle, right wings, left hind wing, gone.

Limneria fusiformis. Type.-Female, yellow label 302. 2nd Coll. Pub. Mus., Quebec. Left antenna at scape, right at apex, gone; median tarsi broken; right hind tarsus and left hind leg at femora, gone.

Limneria guignardi. Type.-Harrington Coll.

Limneria hyalina. Type--Female, yellow label 299. 2nd Coll. Pub. Mus., Quebec. 
Limneria infumata. Type-Female, yellow label 300 . 2nd Coll. Pub. Mus., Quebec. Antennæ and one middle leg broken.

Limneria marginata. Type--Female, yellow label 703. 2nd Coll. Pub. Mus., Quebec. Antennæ broken, the left before the right beyond the middle.

Limneria nigricoxa. Type--Female, yellow label 675 . 2nd Coll. Pub. Mus., Quebec.

Limneria pallipes. Type.-Female, yellow label 304. 2nd Coll. Pub. Mus., Quebec. Antennæ broken beyond middle, left hind leg beyond coxa missing.

Limneria parva. Type.-Female, yellow label 298. 2nd Coll. Pub. Mus., Quebec. One antenna broken at apex; hind legs gone.

Limneria pilosula. Allotype.-Yellow label 1100. 2nd Coll. Pub. Mus., Quebec. Abdomen glued on label, apex of right antenna gone. Type.-Female not located.

Limneria plena. Type.-Female, yellow label 296. 2nd Coll. Pub. Mus., Quebec. Antennæ and right hind tarsus gone.

Limneria ruficornis. Type.-Not in Pub. Mus., Quebec, unless under Limneria dentata Prov.

Limneria ruficoxa. Type.-Female, yellow label 303. 2nd Coll. Pub. Mus., Quebec. Antennæ beyond middle, left hind leg at trochanter, right hind tarsus and ovipositor sheaths, gone.

Limneria rufipes. Type.-Female, yellow label 521 and name label Limneria mellipes Prov. Proved by Provancher catalogue. 2nd Coll. Pub. Mus., Quebec. Antennæ broken near middle.

Limneria sericea. Type.-Not located.

Limneria sessilis. Type--Female, yellow label 348. 2nd Coll. Pub. Mus., Quebec. Antennæ broken before middle.

Linoceras cloutieri. Type.-Female, yellow label 264. 2nd Coll. Pub. Mus., Quebec. Allotype--Male, yellow label 303. 1st Coll. Pub. Mus., Quebec.

Liris magnifica. Type-Male, yellow label 1721. 2nd Coll. Pub. Mus., Quebec.

Liris rugosa. Type--Male, yellow label 1222. 2nd Coll. Pub. Mus., Quebec.

Lonchidia hirta. Type.-Not located. 
Lyda burquei. Type.-Female, yellow' label 126. '1st Coll. Pub. Mus., Quebec. Lacks left flagellum .

Lyda harringtoni. Type.-Female, Harrington Coll.

Lyda quebecensis. Type.-Female, yellow label 504. 2nd Coll. Pub. Mus., Quebec. Lacks antennæ; only one anterior wing left, and that on pin.

Lyroda subita. Type--Not located.

Macrocentrus aciculatus. Type--Female, yellow label 1484. 2nd Coll. Pub. Mus., Quebec.

Macrocentrus longicornis. Type--Male, yellow label 911. 2nd Coll. Pub. Mus., Quebec. Lacks right antenna.

Macrocentrus mellipes. Type-Female, yellow label 571. 2nd Coll. Pub. Mus., Quebec. Lacks antennæ; abdomen loose and dirty.

Macrocentrus pacificus. Type.-Ent. Branch, Dept. Agr., Ottawa. Head gone.

Macrocentrus pectoralis. Type--Male, yellow label 728 . 2nd Coll. Pub. Mus., Quebec. Lacks abdomen and some legs; antennæ incomplete.

Macrophya albipes. Type.-Female, white label 157; yellow label 1676. 2nd Coll. Pub. Mus., Quebec.

Macrophya contaminata. Type--Yellow label 36. 2nd Coll. Pub. Mus., Quebec. Also 2 specimens without labels.

Macrophya crassicornis. Type.-Yellow label 1547. 2nd Coll. Pub. Mus., Quebec.

Megaspilus lucens. Type.-Not in Pub. Mus., Quebec, unless under Proctotrupes flavipes Prov.

Megastylus politus. Type.-Not in Pub. Mus., Quebec, unless under Stilpnus americanus Cress.

Meniscus ashmeadii. Type.-Not located.

Meniscus crevieri. Type.-Not in Pub. Mus., Quebec, unless under. Meniscus scutellaris Cress.

Meniscus marginatus. Type-Female, yellow label 1037. 2nd Coll. Pub. Mus,, Quebec.

Meniscus superbus. Type.-Female, yellow label 418. 2nd Coll. Pub. Mus., Quebec.

Mesochorus areolatus. Type.-Female, yellow label 680 . 
2nd Coll. Pub. Mus., Quebec. ,Left antenna gone at scape; abdomen glued on yellow label.

Mesochorus flaviceps. Type.-Female, yellow label 358. 2nd Coll. Pub. Mus., Quebec.

Mesochorus humeralis. Type.-Female, yellow label 698 . 2nd Coll. Pub. Mus., Quebec.

Mesochorus jucundus. Type.-Female, yellow label 679. 2nd Coll. Pub. Mus., Quebec.

Mesochorus pleuralis. Type.-Female, yellow label 367. 1st Coll. Pub. Mus., Quebec.

Mesochorus politus. Type-Female, yellow label 681 . 2nd Coll. Pub. Mus., Quebec.

Mesochorus rufulus. Type--Female, yellow label 577. 2nd Coll. Pub. Mus., Quebec. Antennæ broken near base; hind legs except left coxa, and abdomen, missing.

Mesochorus saint-cyri. Not in Pub. Mus., Qubeec, unless under Echthrus abdominalis.

Mesochorus truncatus. Type-Female, white label 464, yellow label 1559. 2nd Coll. Pub. Mus., Quebec. On a short pin.

Mesoleius annulatus. Type.-Female, blue label 259(s); yellow label 1242. 2nd Coll. Pub. Mus., Quebec.

Mesoleius antennatus. Type.-Female, yellow label 338 . 2nd Coll. Pub. Mus., Quebec.

Mesoleius canadensis. Type.-Female, yellow label 336. 2nd Coll. Pub. Mus., Quebec.

Mesoleius chicoutimiensis. Type--Female, yellow label 1584. 2nd Coll. Pub. Mus., Quebec. Most of antennæ and legs gone; right hind leg present.

Mesoleius fissus. Type.-Male, yellow label 339. 2nd Coll. Pub. Mus., Quebec. Lacks apices of antennæ.

Mesoleius inflatifrons. Type.-Female, blue label 445. 2nd Coll. Pub. Mus., Quebec.

Mesoleius junctus. Type.-Male, yellow label 936. 2nd Coll. Pub. Mus., Quebec.

Mesoleius mellipes. Type.-Male, yellow label 413. 1st Coll. Pub. Mus., Quebec. 
Mesoleius niger. Type.-Female, yellow label 933. 2nd Coll. Pub. Mus., Quebec.

Mesoleius tardus. Type.-Female, yellow label 337. 2nd Coll. Pub. Mus., Quebec. Abdomen glued on.

Mesoleius telarius. Type.-Female, yellow label 1241, old rose label 74. 2nd Coll. Pub. Mus., Quebec. Right flagellum gone.

Mesoleptus albopleuralis. Type.-Male, yellow label 462 . 2nd Coll. Pub. Mus., Quebec.

Mesoleptus angustus. Type--Male, Harrington Coll., Labelled "Type. Rohw. Gahan."

Mesoleptus annulatipes. Type.-Male, yellow label 1239, name label "Mesoleptus cinctipes Prov." Proved by Provancher's catalogue. 2nd Coll. Pub. Mus., Quebec.

Mesoleptus annulatus. Type--Female, Harrington Coll. Pink label “p. 471" Paratype, yellow label 683. 2nd Coll. Pub. Mus., Quebec.

Mesoleptus barbatus. Type--Male, yellow label 1230 . 2nd Coll. Pub. Mus., Quebec. Provancher mistook sex.

Mesoleptus canaliculatus. Type.-Male, yellow label 322. 2nd Coll. Pub. Mus., Quebec.

Mesoleptus depressus. Type.-Male, yellow label 400 . 2nd Coll. Pub. Mus., Quebec. Lacks hind tarsi.

Mesoleptus erectus. Type-Female, yellow label 399 . 1st Coll. Pub. Mus., Quebec.

Mesoleptus fasciatus. Type.-Not located.

Mesoleptus filiformis. Type.-Male, yellow label 1240. 2nd Coll. Pub. Mus., Quebec. Provancher mistook sex.

Mesoleptus flavicornis Type.-Female, yellow label 464, 2nd Coll. Pub. Mus., Quebec. Lacks flagellum beyond first.

Mesoleptus incompletus. Type.-Not in Pub. Mus., Quebec, unless under Cremastus fusiformis Prov.

Mesoleptus interruptus. Type-Fèmale, yellow label 390. 1st Coll. Pub. Mus., Quebec. Part of antennæ gone.

Mesoleptus lætus. Type--Yellow label 319. 2nd Coll. Pub. Mus., Quebec. Antennæ and abdomen gone.

Mesoleptus largus. Type.-Female, yellow label 1231. 2nd Coll. Pub. Mus., Quebec. 
Mesoleptus laurentianus. Type--Male, yellow label 394. 1st Coll. Pub. Mus., Quebec. Most of left flagellum, left hind leg, apical joints of right hind tarsi gone.

Mesoleptus longipes. Type.-Not in Pub. Mus., Quebec, unless under $M$. moveni Prov.

Mesoleptus lucens. Type--Male, yellow label 318. 2nd Coll. Pub. Mus., Quebec.

Mesoleptus maculatus. Type.-Not in Pub. Mus., Quebec, unless under Exyston clavatus Prov.

Mesoleptus moyeni. Type-Female, yellow label 315. 2nd Coll. Pub. Mus., Quebec.

Mesoleptus nigricornis. Type--Female, Harrington Coll.

Mesoleptus perditus. Type--Male, blue label 165(s); yellow label 1257. 2nd Coll. Pub. Mus., Quebec. Lacks apex of left antenna.

Mesoleptus rhopalocerus. Type.-Male, yellow label 1561. 2nd Coll. Pub. Mus., Quebec.

Mesoleptus rufipes. Type.-Female, yellow label 406. 1st Coll. Pub. Mus., Quebec.

Mesoleptus rufomixtus. Allotype--Male, Harrington Coll. Type.-Female not located.

Mesoleptus rufulus. Type,-Female, yellow label 455 . 2nd Coll. Pub. Mus., Quebec. Lacks apices of tarsi except one; abdomen glued on yellow label.

Mesoleptus sancti-hyacinthi. Type.-Not in Pub. Mus., Quebec, unless under Mesoleptus inceptus Cress.

Mesoleptus seminiger. Type--Female, yellow label 467. 2nd Coll. Pub. Mus., Quebec. Lacks flagella. Allotype.-Male, yellow label 401... 1st Coll. Pub. Mus., Quebec.

Mesoleptus sericeus. Type.-Female, yellow label 483. 2nd Coll. Pub. Mus., Quebec. Apices of flagella wanting; abdomen loose.

Mesoleptus uniformis. Type.-Female, yellow label 402. 1st Coll. Pub. Mus., Quebec. Cocoon on pin. Labeled Campoplex unicolor. Proved by Provancher's Catalogue, Provancher mistook sex.

(To be continued.) 
LECTOTYPES OF HYMENOPTERA (E'XCEPT APOIDEA) DESCRIBED BY ABBE PROVANCHER.

BY A. B. GAHAN AND S, A. ROHWER, WASHINGTON, D, C.

(Continued from Vol..L, page 33.)

Mesoleptus variabilis. (Faun. 1883, p. 759, not Nat. Can. 7, p. 115). Type.-Female, yellow label 682. 2nd Coll. Pub. Mus., Quebec.

Mesoleptus variabilis. (Nat. Can. 7, p. 115, not Fauna 1883 , p. 795). Type.-Male, yellow label 1080 , name label Mesoleptus muliebris Cress. 2nd Coll. Pub. Mus., Quebec. Apical joints of hind tarsi gone.

Mesostenus albicoxus. See Phygadeuon.

Mesostenus albifacies. Type.-Female, blue label 553; yellow label 1583. 2nd Coll. Pub. Mus., Quebec. Fair, somewhat oily.

Mesostenus apicalis. Type.-Not in Pub. Mus., Quebec, unless under Ichneumon finitimus Cress.

Mesostenus armatus. Type.-Female, Harrington Coll. Pink label "P. 416."

Mesostenus brevipennis. Type. - Not in Pub. Mus., Quebec, unless under Mesostenus promptus Cress. 'This synonymy indicated by Provancher's annotated copy of Fauna.

Mesostenus collinus. Type.-Not in Pub. Mus., Quebec, unless under Cryptus eburneifrons Prov.

Mesostenus flavipes. Type.-Male, yellow label 459. 2nd Coll. Pub. Mus., Quebec. Fair.

Mesostenus jocosus. Type.-Female, yellow label 304. 1st Coll. Pub. Mus., Quebec. Lacks apices of antennæ.

Mesostenus latigaster. 'Type.-Female, Harrington Coll. Pink label "P. 407." Abdomen broken off and when glued on covered propodeum.

Mesostenus longicornis. Type. - Not in Pub. Mus., Quebec,, unless under Mesoleptus moveni Prov.

Mesostenus nitidus. Not Grav. See Phygadeuon nitidulus.

Mesostenus nobilis. Type--Female, yellow label 1049. March, 1918 
2nd Coll. Pub. Mus., Quebec. Left antenna gone and apices of right wanting.

Mesostenus pallipes. Type.-Not in Pub. Mus., Quebec, unless under Mesoleptus triangularis Cress.

Mesostenus pluricinctus. Type--Harrington Coll. Pink label "P. 462." Davis has labeled this Diacritus muliebris Cress.

Mesostenus ruficornis. Type.-Not in Pub. Mus., Quebec, unless under Phygadeuon nitidulus Prov.

Mesostenus ruficoxus. Type.-Not located. =provancheri D. T.

Mesostenus rufocinctus. Type.-Not located.

Mesostenus sagax. Type-Female, yellow label 522. 2nd Coll. Pub. Mus., Quebec. Lacks left antenna and apex of right.

Mesostenus saint-cyri. Type.-Not in Pub. Mus., Quebec, unless under Echthrus abdominalis Cress.

Mesostenus tarsatus. Type.-Not in Pub. Mus., Quebec, unless under Cryptus americanus Cress.

Metacolus conicus. Type-Female, yellow label 1365. 2nd Coll. Pub. Mus., Quebec. Fair.

Meteorus gracilis. Type.-Female, yellow label 1264. 2nd Coll. Pub. Mus., Quebec. Poorly mounted on tag. Antennæ and right hind leg missing.

Meteorus incompletus. Type.-Male, yellow label 1283. 2nd Coll. Pub. Mus., Quebec. Poorly pinned. Antennæ broken.

Meteorus politus. Type.-Female, yellow label 1266. 2nd Coll. Pub. Mus., Quebec. Badly pinned.

Meteorus robustus. Type.-Female, yellow label 1265. 2nd Coll. Pub. Mus., Quebec. Poorly pinned, one antenna beyond middle and hind legs at coxæ, gone.

Microctonus laticeps. Type--Female, yellow label 1286; blue label 616. 2nd Coll. Pub. Mus., Quebec. Right antenna at 4th joint, front legs, right hind leg missing.

Meteorus vigilax. Type.-Probably male, yellow label 565 . 2nd Coll. Pub. Mus., Quebec. Abdomen gone. Allotype, female without labels.

Microdus bicolor. Type.-Female, yellow label 905. 2nd Coll. Pub. Mus., Quebec. 
Microdus dispar. Type-Female, yellow label 1256(s). 2nd Coll. Pub. Mus., Quebec. Poorly tag-mounted.

Microdus nigricoxus. Type.-Female, blue label 656; yellow label 1572. 2nd Coll. Pub. Mus., Quebec.

Microdus quebecensis. Type-Female, yellow label 581. 2nd Coll. Pub. Mus., Quebec.

Microgaster auripes. Type.-Female, blue label 673; yellow label 590. 2nd Coll. Pub. Mus., Quebec. One antenna broken at middle.

Microgaster brevicaudus. Type--Female, yellow label 1167. 2nd Coll. Pub. Mus., Quebec. One antenna broken at tip.

Microgaster crenulatus. Type--Male, yellow label 1577. 2nd Coll. Pub. Mus., Quebec. Left fore leg and left hind leg missing.

Microgaster lateralis. Type--Yellow label 1306. 2nd Coll. Pub. Mus., Quebec. Antennæ, wings on right side and abdomen missing.

Microgaster melligaster. Type.-Female, yellow label 1301. 2nd Coll. Pub. Mus., Quebec. Antennæ broken at tip.

Microgaster 4-dentatus. Male, blue label 45, yellow label 1271. 2nd Coll. Pub. Mus., Quebec.

Microgaster rubricoxus. Type--Male, yellow label 1576. 2nd Coll. Pub. Mus., Quebec. Antennæ and right hind leg broken.

Micromelus nigricornis. Type--Yellow label 942. 2nd Coll. Pub. Mus., Quebec. Fair.

Mictropis nebulosa. Type--Harrington Coll.

Monedula nigrifrons. Type.-Female, yellow label 1616 . 2nd Coll. Pub. Mus., Quebec.

Monedula parata. Type.-Female, yellow label 1617. 2nd Coll. Pub. Mus., Quebec.

Monobia bicolor. Type.-Female, white label 61(s); yellow label 1629. 2nd Coll. Pub. Mus., Quebec.

Monodontomerus viridæneus. Type.-Not in Pub. Mus., Quebec, unless under Oligostenus stigma Fabr.

Monophadnus pallipes. Type.-Female, yellow label 1675 . 2nd Coll. Pub. Mus., Quebec.

Mutilla canadensis. Type.-Not located.

Myrmica incompleta. Type.-Not located. 
Nematopodius coxatus. Type.-Not in Pub. Mus., Quebec, unless under Cryptus americanus Cress.

Nematus decoratus. Yellow label 1541. 2nd Coll. Pub. Mus. Quebec.

Nematus fulvicrus. Type.-Yellow label 485. 2nd Coll. Pub. Mus., Quebec.

Nematus tetraopsis. Type.-Yellow label 1673. 2nd Coll. Mus. Parlement, Quebec.

Neuropenes ovalis. Type-See Introduction. (Aphidiinæ.)

Neuroterus crassitelus. Type.-White label "Cap Rouge, Canada;" yellow label 934. 2nd Coll. Pub. Mus., Quebec. Bad condition. Cynips (Neuroterus) crassitelus-under Cynips in Provancher's catalogue.

Neuroterus terminalis. Type.-White label 114; white label 72(s); yellow label 1593. 2nd Coll. Pub. Mus., Quebec.

Nomia compacta. Type.-Not in Pub. Mus., Quebec.

Nototrachis canadensis. Type.-Female, yellow label 275. 2nd Coll. Pub. Mus., Quebec. Antennæ at scape and front legs missing.

Nysson nigripes. Type.-Male, yellow label 1429. 2nd Coll. Pub. Mus., Quebec.

Odontomerus canadensis. Type-Female, yellow label 426. 2nd Coll. Pub. Mus., Quebec. Lacks right antenna. Male, allotype, without labels.

Odynerus antillarum. Type.-Not located.

Odynerus bimaculatus. Type.-Female, yellow label 1692 . 2nd Coll. Pub. Mus., Quebec.

Odynerus robustus. Type.-Female, yellow label 1708 . 2nd Coll. Pub. Mus., Quebec.

Odynerus tricolor. Type.-Male, white label 182, yellow label 1694. 2nd Coll. Pub. Mus., Quebec.

Odynerus truncatus. Type-Male, yellow label 1693. 2nd Coll. Pub. Mus., Quebec.

Oenone sericea. Type.-Female. 2nd Coll. Pub. Mus., Quebec. Poorly tag mounted, right antenna broken at tip.

Oligostenus bimaculatus. Type--Harrington Coll.

Ophion nigrovarius. Type--Female, yellow label 273. 2nd Coll. Pub. Mus., Quebec. Antennæ, left hind leg beyond 
femora, right hind tarsus in part gone; abdomen broken off but stuck on pin below label. Allotype-Male, yellow label 322. 1st Coll. Pub. Mus., Quebec.

Opius cinctus. Type--See Introduction.

Opius macrocephalus. Type.-Not in Pub. Mus., Quebec, unless under under Alysia rudibunda Say.

Opius mellipes. Type. $-\mathrm{n}$. n. for $O$. pallipes Prov.

Opius nanus. Type--See Introduction.

Opius niger. Type.-See Introduction.

Opius pallipes. See Introduction.

Opius ruficeps. Type--See Introduction.

Opius variabilis. Type.-See Introduction.

Orgilus detectus. Type.-Femate, blue label 680 , yellow label 1573. 2nd Coll. Pub. Mus., Quebec.

Ormyrus bruneipes. Type.-Female, yellow label 1358. 2nd Coll. Pub. Mus., Quebec. Fair.

Orthocentrus abdominalis. Type-Female, yellow -label 357. 2nd Coll. Pub. Mus. Quebec.

Orthocentrus albofasciatus. Type--Male, yellow label 935. 2nd Coll. Pub. Mus., Quebec. Lacks apices of antennæ.

Orthocentrus canadensis. Type--Male, yellow label 356 . 2nd Coll. Pub. Mus., Quebec.

Orthocentrus carinatus. Type--Male, yellow label 518. 2nd Coll. Pub. Mus., Quebec. Apices of antennæ gone.

Orthocentrus lucens. Type.-Not located.

Orthocentrus nigricoxus. Type--Not lócated. Probably returned to collector.

Orthocentrus pilifrons. Type--Yellow label 355. 2nd Coll. Pub. Mus., Quebec. Lacks antennæ, some legs and abdomen.

Oxybelus brodiei. Type.-Female, yellow label 1041. 2nd Coll. Pub. Mus., Quebec. Double mounted and with fly as prey. Apex of right fore wing gone.

Oxylabis spinosus. Type.-Blue label 759 ; yellow label 943. 2nd Coll. Pub. Mus., Quebec.

Pachyprotasus delta. Type--Female, yellow label 496. 2nd Coll. Pub. Mus., Quebec. Right flagellum gone. 
Pammegischia burquei. Type.-Female, yellow label 507 . 2nd Coll. Pub. Mus., Quebec.

Paniscus albotarsatus. Type--Male, yellow label 288. 2nd Coll. Pub. Mus., Quebec.

Paniscus albovariegatus. Type-Male, yellow label 287. 2nd Coll. Pub. Mus., Quebec. Antennæ and abdomen at apex broken off.

Paniscus appendiculatus. Type- -Not in Pub. Mus., Quebec, unless under name, Paniscus geminatus Say.

Paniscus quebecensis. Type.-Not in Pub. Mus., Quebec̄, unless under name Exetastes suaveolens Walsh

Pantoclis inermis. Type.-Yellow label 1119. 2nd Coll. Pub. Mus., Quebec. Fair.

Paphagus rugosus. - Type.-Yellow label 1383. 2nd Coll. Pub. Mus., Quebec. Badly mounted.

Paramesius longicornis. Type--Yellow label 950. 2nd Coll. Pub. Mus., Quebec. Fair.

Pelopæus argentipilis. Type.-Not located.

Periclistus obliquus. Type.-White label 156; white labèl 74(s); yellow label 1594. 2nd Coll. Pub. Mus., Quebec.

Perilampus aciculatus. Type.-Blue label $767(\mathrm{~s})$; yellow label 1359. 2nd Coll. Pub. Mus., Quebec. Antennæ and abdomen gone.

Perilampus lævis. Type--Harrington Coll. ParatypeYellow label 932. 2nd Coll. Pub. Mus., Quebec. Badly mounted.

Perilampus stygicus. Type--Yellow label 1599. 2nd Coll. Pub. Mus., Quebec. Fair.

Perilitus incompletus. See Meteorus.

Perilitus mellinus. Type.-Female, yellow label 727. 2nd' Coll. Pub. Mus., Quebec. Antennæ, median tarsi, right hind leg at coxæ, left hind tarsi broken.

Perilitus nigritus. Type--Male, yellow label 1579. 2nd Coll. Pub. Mus., Quebec. Tag mounted. Right antenna entirely, left beyond middle, left posterior wing, median and hind leg on left and right hind tarsi missing.

Pezomachus niger. Type.-Female, white label 34(s); yellow label 1492. 2nd Coll. Pub. Mus., Quebec. Fair.

(To be continued) 
LECTOTYPES OF THE SPECIES OF HYMENOPTERA (EXCEPT APOIDEA) DESCRIBED BY ABBÉ PROVANCHER.

BY A. B. GAHAN AND S. A. ROHWER, WASHINGTON, D. C.

(Continued from page 106.)

Pezomachus quebecensis. Type.-Not in Pub. Mus., Quebec, unless under Pezomachus canadensis Cress.

Pezomachus sulcatus. Type.-Not located. Probably in Harrington collection.

Phænocarpa rubriceps. Type--Male, yellow label 1052. 2nd Coll. Pub. Mus., Quebec.

Phæogenes annulatipes. Type--Harrington Coll.

Phæogenes aterrimus. Type--Female, yellow label 435 . 2nd Coll. Pub. Mus., Quebec. Left antenna at apex and left hind tarsus broken.

Phæogenes crassitelus. Type--Female, yellow label 1196. 2nd Coll. Pub. Mus., Quebec. Ovipositor and sheath btoken off at apex of abdomen.

Phæogenes falardeaui. Type--Female, yellow label 666 . 2nd Coll. Pub. Mus., Quebec. Allotype without labels.

Phæogenes gaspesianus. Type.-Female, yellow label 664 . 2nd Coll. Pub. Mus., Quebec. Right antenna broken at 5th joint.

Phæogenes huarti. Type.-Female, yellow label 328. 2nd Coll. Pub. Mus., Quebec. Lacks abdomen.

Phæogenes indistinctus. Type--Male, Harrington Coll. Pink label "P. 409." Lacks apex of left antenna. Labeled by Davis = "Phygadeuon."

Phæogenes mellinus. Type-Female, yellow label 719 . 2nd Coll. Pub. Mus., Quebec. Some verdigris.

Phæogenes nigricornis. Type.-Male, yellow label 988. 2nd Coll. Pub. Mus., Quebec.

Phæogenes orbus. Type.-Male, yellow label 514. 2nd Coll. Pub. Mus., Quebec. Antennæ broken at tip.

Phæogenes pinguis. Type.-Female, Harrington Coll. Head missing.

Phæogenes recticaudus. Type. - Female, yellow label 1197. 2nd Coll. Pub. Mus., Quebec. Abdomen broken off and glued on label.

April, 1918 
Phæogenes recticornis. Type.-Female, yellow label 1200 , blue label 15. 2nd Coll. Pub. Mus., Quebec. Right antenna at apex, right fore wing, hind wings, legs on left side, missing; abdomen broken off and glued on label.

Phæogenes sectus. Type.-Male, white label 439; yellow label 1552. 2nd Coll. Pub. Mus., Quebec. Lacks left antenna and left hind leg. On short pin.

Phæogenes tuberculifer. Type--Male (?), yellow label 979. 2nd Coll. Pub. Mus., Quebec.

Phanerotoma fasciata. Type--Yellow label 599. 2nd Coll. Pub. Mus., Quebec. Abdomen and antennæ beyond third joint missing.

Phasgonophora elegans. Type. - Harrington collection. Paratype.-Blue label 73(s); yellow label 1341. 2nd Coll. Pub. Mus., Quebec.

Philanthus harringtoni. Type.-Not located. Probably returned to collector.

Photopsis canadensis. Type.-Blue label 690; yellow label 1400. 2nd Coll. Pub. Mus, Quebec.

Phygadeuon abdominalis. Type.-Female, yellow label 218. 2nd Coll. Pub. Mus., Quebec. Some verdigris. Allotype.Yellow label 271. 1st Coll. Pub. Mus., Quebec.

Phygadeuon acaudus. Type.-Female, yellow label 1017. 2nd Coll. Pub. Mus., Quebec. Complete but pin obliterates some of the characters.

Phygadeuon aciculatus. Type.-Male, yellow label 665. 2nd Coll. Pub. Mus., Quebec.

Phygadeuon albicoxus. Type.-Male, yellow label 269 , 2nd Coll. Pub. Mus., Quebec.

Phygadeuon alternans. Type.-Yellow label 669. 2nd Coll. Pub. Mus., Quebec. Only thorax and coxæ present.

Phygadeuon annulatus. (Name preoccupied.) See Phygadeuon fusiformis.

Phygadeuon apicatus. Type.-Not in Pub. Mus., Quebec, unless under Ichneumon velox Cress.

Phygadeuon ater. Type.-Not in Pub. Mus., Quebec, unless under Ichneumon helvipes Cress. 
Phygadeuon attenuatus. Type-Female, yellow label 1048. 2nd Coll. Pub. Mus., Quebec.

Phygadeuon autumnalis. Type.-Yellow label 640. 2nd Coll. Pub. Mus., Quebec. Thorax and part of legs on pin, rest missing.

Phygadeuon brevicaudus. Type.-Female, yellow label 1181. 2nd Coll. Pub. Mus., Quebec.

Phygadeuon capitalis. Type--Male, Harrington Coll. Pink label "P. 413."

Phygadeuon caudatus. Type--Female, yellow label 265. 1st Coll. Pub. Mus., Quebec.

Phygadeuon cephalicus. Type.-Male, yellow label 663 . 2nd Coll. Pub. Mus., Quebec.

Phygadeuon constrictus. Type--Yellow label 718. 2nd Coll. Pub. Mus., Quebec. Lacks abdomen and tarsi.

Phygadeuon cornutus. Type.-Female, yellow label 938. 2nd Coll. Pub. Mus., Quebec.

Phygadeuon crassipes. Type--Female, yellow label 276 . 1st Coll. Pub. Mus., Quebec.

Phygadeuon cressoni. Type.-Not in Pub. Mus., Quebec. unless under-Ichneumon velox Cress.

Phygadeuon curticrus. Type--Female, yellow label 1558. 2nd Coll. Pub. Mus., Quebec.

Phygadeuon dorsalis. Type.-Not in Pub. Mus., Quebec, unless under Ichneumon humilis Prov.

Phygadeuon dubius. Type.-Not in Pub. Mus., Quebec, unless under Phygadeuon pubescens Prov.

Phygadeuon electus. Type.-Male, white label 1; yellow label 1117. 2nd Coll. Pub. Mus., Quebec. Lacks apices of antennæ. Provancher mistook sex.

Phygadeuon excavatus. Type--Not located.

Phygadeuon fasciatus. Type--In Pub. Mus., Quebec, with name label. Other data not obtained.

Phygadeuon fraterculus. Type--Female, Harrington Coll. Pink label "P. 404." Lacks left hind leg below coxa and right hind tibia and tarsi.

Phygadeuon fusiformis. Type--Female, Harrington Coll. Pink label "P. 391." Lacks left flagellum. 
Phygadeuon goddessii. Type.--Not located. Probably returned to collector.

Phygadeuon gracilicornis. Type. - Female, Harrington Coll. Left antenna broken.

Phygadeuon guignardi. Type.-Female, yellow label 1178. 2nd Coll. Pub. Mus., Quebec.

Phygadeuon hilaris. Type.-Not in Pub. Mus., Quebec, unless under Phøogenes helvus. Cress.

Phygadeuon impressus. (Nat. Can. Vol. 7, p. 212) Type.Male, yellow label 213. 2nd Coll. Pub. Mus., Quebec.

Phygadeuon impressus. (Nat. Can. Vol. 6, p. 281) Type. - Not in Coll. unless under name Platylabus thoracicus Cress.

Phygadeuon inflatus. Type- - Not located. Female, yellow label 221 bearing this name label in 2nd Coll. Pub. Mus., Quebec, cannot be type.

Phygadeuon inhabilis. Type--Female, yellow label 205. 2nd Coll. Pub. Mus., Quebec.

Phygadeuon insignis. Type.-Not in Pub. Mus., Quebec, unless under Phcoogenes herbus Cress.

Phygadeuon jocosus. Type.-Female, old rose label 90, yellow label 1180. 2nd Coll. Pub. Mus., Quebec.

Phygadeuon lavoiei. Type--Female, yellow label 660 . 2nd Coll. Pub. Mus., Quebec.

Phygadeuon lechevallieri. Type.-Yellow label 692. 2nd Coll. Pub. Mus., Quebec. Lacks antennæ, legs, left wings and abdomen.

Phygadeuon longicornis. Type. - Female, Harrington Coll. Pink label "P. 388." Lacks apex of right antenna.

Phygadeuon lucens. Type.-Female, yellow label 270. 1st Coll. Pub. Mus., Quebec. Lacks antennæ.

Phygadeuon maculatus. Type--Female, yellow label 206. 2nd Coll. Pub. Mus., Quebec. Lacks antennæ.

Phygadeuon marginatus. Type--Male, Harrington Coll. One antenna and apical half of other missing.

Phygadeuon maturus. Type.-Female, yellow label 453 . 2nd Coll. Pub. Mus., Quebec. Apices of antennæ and left fore wing gore. 
Phygadeuon mignaulti. Type.-Female, yellow label 661 . 2nd Coll. Pub. Mus., Quebec. Lacks right antenna.

Phygadeuon mucronatus. Type.-Female, yellow label 611. 1st Coll. Pub. Mus., Quebec.

Phygadeuon niger. Type.-Not in Pub. Mus., Quebec, unless under Ichneumon extrematatis Cress.

Phygadeuon nigriceps. Type.-Female, old rose label 40, yellow label 1179. 2nd Coll. Pub. Mus., Quebec.

Phygadeuon nitidulus. Type.-Male, yellow label 262 . 1st Coll. Pub. Mus., Quebec.

Phygadeuon occidentalis. Type-Female, yellow label 263. 1st Coll. Pub. Mus., Quebec.

Phygadeuon orbitalis. Type--Male, yellow label 513. 2nd Coll. Pub. Mus., Quebec.

(To be continued)

\section{SOME SOUTH AMERICAN BEES.}

BY T. D, A. COCKERELL, BOULDER, COLORADO.

\section{Colletes chubutensis, sp. $\mathrm{n}$.}

$\sigma^{7}$.- Length about $10 \mathrm{~mm}$., anterior wing $7.5 \mathrm{~mm}$.; head, thorax and legs black, abdomen obscure steel-blue; head, seen from in front, forming roughly an equilateral triangle, the vertex being very broad; malar space extremely long, fully twice width of mandibles at base; mandibles red at apex; labrum rather weakly plicate basally; clypeus shining, very sparsely punctured, with a broad, band-like median depression; antennæ black, long, reaching metathorax; third joint 320 microns long, fourth 480, fifth 450 ; mesothorax and scutellum dullish, not polished; area of metathorax smooth and shining, with a sub-basal, transverse ridge, above which, in the middle, is a small pit; hair of head and thorax very long and abundant, white on face, cheeks and under side of thorax; sides of face with black hairs; hair of upper part of head and thorax with blackish intermixed, the whole appearing grey; tegulæ piceous; wings hyaline, faintly dusky, nervures and stigma dark fuscous; second s.m. extremely broad, receiving first r.n. in middle; legs with white hair, extremely long on anterior femora posteriorly; abdomen shining, hardly punctured; hind margins of segments narrowly brownish, without hair-bands; dorsal surface 
LECTOTYPES OF THE SPECIES OF HYMENOPTERA (EXCEPT APOIDEA) DESCRIBED BY ABBE PROVANCHER.

BY A. B. GAHAN AND S. A. ROHWER, WASHINGTON, D. C.

(Continued from page 137.)

Phygadeuon ovalis. Type.-Female, yellow label 211. 2nd Coll. Pub. Mus., Quebec.

Phygadeuon pallicoxus. Type--Yellow label 457. 2nd Coll. Pub. Mus., Quebec. Thorax, legs and wings on pin, rest missing.

Phygadeuon parallelus. Type--Male, yellow label 939. 2nd Coll. Pub. Mus., Quebec.

Phygadeuon planus. Type.-Female, yellow label 223. 2nd Coll, Pub. Mus., Quebec.

Phygadeuon pubescens. Tỳpe.-Male, yellow label 273. 1st Coll. Pub. Mus., Quebec.

Phygadeuon rectus. Type.-Male, yellow label 204. 2nd Coll. Pub. Mus., Quebec.

Phygadeuon robustus. Type--Female, yellow label 458 . 2nd Coll. Pub. Mus., Quebec.

Phygadeuon rotundiceps. Type.-Female, yellow label 220. 2nd Coll. Pub. Mus., Quebec.

Phygadeuon rubricus. Type--Female, yellow label 701. 2nd Coll. Pub. Mus., Quebec. Extreme apex of left antenna gone. Phygadeuon rubrocinctus. Type--Female, yellow label 259. 1st Cell. Pub. Mus., Quebec. Somewhat oily.

Phygadeuon ruficornis. Type--Male, yellow label 222. 2nd Coll. Pub. Mus., Quebec.

Phygadeuon rufipes. Type.-Not in Pub. Mus., Quebec, unless under Platylabus lineolatus. Prov.

Phygadeuon segnis. Type.-Female, yellow, label 267. 1st Coll. Pub: Mus., Quebec.

Phygadeuon signatus. Type.-Female, yellow label 258. 1st Coll. Pub. Mus., Quebec.

Phygadeuon similaris. Type.-Male, Harrington Coll. Pink label "P. 399." Antennæ gone beyond 3rd joint; mounted so propodeum is hard to see.

May, 1918 
Phygadeuon subspinosus. Type--Male, yellow label 668 . 2nd Coll. Pub. Mus., Quebec.

Phygadeuon tegularis. Type.-Not in Pub. Mus., Quebec, unless under Phygadeuon alacris Cress.

Phygadeuon terminalis. Type. - Not in Pub. Mus., Quebec, unless under Ichneumon caudatus.

Phygadeuon terminatus. Type--Female, yellow label 437. 2nd Coll. Pub. Mus., Quebec. Abdomen off but glued on label.

Phygadeuon 3-annulatus. Type.-Female, yellow label 981. 2nd Coll. Pub. Mus., Quebec. Abdomen and apices of antennæ gone.

Phygadeuon truncatus. Type--Not located.

Phylax niger. Type.-Ent. Branch, Dept. Agr., Ottawa.

Phylloecus bicinctus. Type.-Female, yellow label 180 . 1st Coll. Pub. Mus., Quebec.

Phytodietus elegans. Type.-Female, yellow label 1653. 2nd Coll. Pub. Mus., Quebec.

Phytodietus ornatus. Type--Female, yellow label 1654 . 2nd Coll. Pub. Mus,, Quebec.

Phytodietus superbus. Type.-Female, yellow label 1652. 2nd Coll. Pub. Mus., Quebec. Lacks antenna beyond annulus.

Phytodietus zonatus. Type.-Yellow label 514. 1st Coll. Pub. Mus., Quebec. Lacks abdomen and right antenna.

Pimpla æqualis. Type.-Female, yellow label 715. 2nd Coll. Pub. Mus., Quebec. Lacks apex of left antenna.

Pimpla hirticauda. Type.-Female, yellow label 1252. 2nd Coll. Pub. Mus, Quebec.

Pimpla 4-cingulatus. Type-Female, yellow label 714 . 2nd Coll. Pub. Mus., Quebec.

Platygaster aneurus. Type.-Yellow label 1329. 2nd Coll. Pub. Mus., Quebec. Fair.

Platylabus aciculatus. Type--Yellow label 1187. 2nd Coll. Pub. Mus., Quebec. Lacks one hind leg beyond femora.

Platylabus cincticornis. Type--Female, yellow label 1188. 2nd Coll. Pub. Mus., Quebec.

Platylabus crassicornis. Type.-Female, yellow label 1186.

2nd Coll. Pub. Mus., Quebec. 
Platylabus lineolatus. Type-Female, yellow label 686 . 2nd Coll. Pub. Mus., Quebec.

Platylabus magnificus. Type.-Female, old rose label 66 , yellow label 1184. 2nd Coll. Pub. Mus., Quebec.

Platylabus mitralis. Type.-Female, yellow label 1185. 2nd Coll. Pub. Mus., Quebec.

Platylabus ornatus. Type.-Female, yellow label 244. 1st Coll. Pub. Mus., Quebec. Head missing.

Platylabus 4-carinatus. Type-Male, yellow label 443. 2nd Coll. Pub. Mus., Quebec. Antennæ gone.

Platylabus rubricapensis. Type.-Female, yellow label 717. 2nd Coll. Pub. Mus., Quebec.

Platylabus ruficornis. Type. - Male, Harrington Coll. Pink label "P. 417." Lacks apices of antennæ. Allotype.Female, yellow label 1211; blue-gray label 418. 2nd Coll. Pub. Mus., Quebec.

Platylabus scutellatus. Type.-Yellow label 195. 2nd Coll. Pub. Mus., Quebec.

Platylabus signatus. Type.-Not located.

Platymischus torquatus. Type.-Old rose label 53; yellow label 1337. 2nd Coll. Pub. Mus., Quebec. Badly glued.

Plectiscus gracilis. Type.-Female, yellow label 369. 1st Coll. Pub. Mus., Quebec. Good, but part of wings on pin.

Plectiscus niger. Type.-Male, yellow label 723. 2nd Coll. Pub. Mus., Quebec. Part of antennæ gone.

Podogaster radiolatus. Type.-Female, yellow label 482 . 2nd Coll. Pub. Mus., Quebec. Antennæ broken at tip.

Podogaster sulcatus. Type--Yellow label 1225. 2nd Coll. Pub. Mus., Quebec. Right antenna at scape and abdomen gone.

Polistes anaheimensis. Type-Cat. No. 1978, U. S. Nat. Mus.

Polyblastus annulicornis. Type.-Male, yellow label 97, also yellow label 1243. 2nd Coll. Pub. Mus., Quebec. Provancher mistook sex.

Polyblastus decoratus. Type.-Male, blue label $486 . \quad$ 2nd Coll. Pub. Mus., Quebec.

Polyblastus dilatatus. Type.-Female, yellow label 334 . 2nd Coll. Pub. Mus., Quebec. Lacks antennæ. 
Polyblastus gaspesianus. Type.- Not located.

Polyblastus inornatus. Type--Female, blue label 566 . 2nd Coll. Pub. Mus., Quebec.

Polyblastus quebecensis. Type--Female, yellow label 427. 1st Coll. Pub. Mus., Quebec.

Polysphincta acuta. Type--Female, yellow label 393. 2nd Coll. Pub. Mus., Quebec. Lacks abdomen, apices of antennæ and apical joints of hind tarsi.

Polysphincta bruneti. Type.-Female, yellow label 710 . 2nd Coll. Pub. Mus., Quebec.

Polysphincta cingulata. Type--Yellow label 486. 1st Coll. Pub. Mus., Quebec. Only thorax, left wings and a set of legs present.

Polysphincta pleuralis. Type.-Not in Pub. Mus., Quebec, unless under Bassus pulchripes Prov.

Polysphincta rubricapensis. Type-Female, yellow label 403. 2nd Coll. Pub. Mus., Quebec.

Polysphincta rufopectus. Type.-Not in Pub. Mus., Quebec, unless under P. limata Cress.

Polysphincta vicina. Type.-Male, unlabeled. 1st Coll. Pub. Mus., Quebec. Lacks flagellum. Stands with female bearing yellow label 394 .

Pompilus apicatus. Type.-Female, yellow label 769 . 2ne Coll. Pub. Mus., Quebec. Lacks left flagellum.

Pompilus castaneus. Type.-Male, yellow label 774 . Name label Pompilus argenteus Cress. 2nd Coll. Pub. Mus., Quebec. Lacks apices of antennæ. Proved by Prov. Catalog.

Pompilus coquilletti. Type.-Male, Cat. No. 1980, U. S. Nat. Mus.

Pompilus griseus. Type.-Male, yellow label 1011. 2nd Coll. Pub. Mus., Quebec. Lacks antennæ, rather dirty.

Porizon albipes. Type--Male, yellow labels 1554 (Prov.) and 150 (not Prov.). 2nd Coll. Pub. Mus., Quebec. Left antenna gone. Provancher mistook sex.

Porizon angulare. Type.-Male, yellow label 452. 2nd Coll. Pub. Mus., Quebec. Left antenna broken near base.

Porizon boreale. Type.-Female, yellow label 370. 1st Coll. Pub. Mus., Quebec. Head and left fore leg missing. 
Porizon californicum. Type.-Male, small, square, white labels 34 and 2 (s); yellow label 1480. 2nd Coll. Pub. Mus., Quebec. Right antenna at scape, left at middle, and left hind wing, gone.

Porizon elongatum. Type.-Male, yellow label 1226. 2nd Coll. Pub. Mus., Quebec.

Porizon rugosum. Type.-Male, yellow label 445. 2nd Coll. Pub. Mus., Quebec. Antennæ broken near middle.

Posocentrus huarti. See Phoogenes huarti.

Praon simulans. Type.-See introduction (Aphidiinæ).

Priononyx canadensis. Type.-Male, yellow label 1070. 2nd Coll. Pub. Mus., Quebec.

Proctotrupes flavipes. Type. - Yellow label 618. 2nd Coll. Pub. Mus., Quebec.

Proctotrupes rufigaster. Type--Female, yellow label 641. 2nd Coll. Pub. Mus., Quebec.

Prosapha hyalina. Type.-Male, yellow label 1586. 2nd Coll. Pub. Mus., Quebec. Right antenna at scape, wings on right and legs on right, except fore and hind femora, gone.

Pteromalus acutus. Type--Yellow label 922. 2nd Coll. Pub. Mus., Quebec. Badly glued.

Pteromalus nigricornis. Type.-Yellow label 1602. 2nd Coll. Pub. Mus., Quebec. Badly glued.

Pteromalus pieridis. Type.-Yellow label 628. 2nd Coll. Pub. Mus., Quebec.

Pyracmon annulatum. Type--Yellow label 524. 2nd Coll. Pub. Mus., Quebec. Left antenna at scape, right at middle, posterior legs except right coxa, and abdomen gone.

Pyracmon incompletum. Type--Female, yellow label 1224. 2nd Coll. Pub. Mus., Quebec.

Pyracmon macrocephalum. Type-Female, yellow label 305. 2nd Coll. Pub. Mus., Quebec. Antennæ gone,

Pyracmon rufum. Type.-Female, yellow label 1031. 2nd Coll. Pub. Mus., Quebec. Lacks apex of left flagellum.

Radiolaria clavata. Type,-Blue label $742(\mathrm{~s})$; yellow label 1290. 2nd Coll. Pub. Mus., Quebec. Tag-mounted, right antenna broken at tip.

Rhogas quebecensis. Type.-Female, yellow label 545. 2nd Coll. Pub. Mus., Quebec. 
Rhogas rugosulus. Type.-Female, yellow label 1567. 2nd Coll. Pub. Mus., Quebec. Lacks ovipositor and most of legs.

Rhogas sancti-hyacinthi. Type.-Female, yellow label 546. 2nd Coll. Pub. Mus., Quebec. Antennæ broken at tips.

Rhopalicus pallipes. Type.-Not located.

Rhopalophorus. See Eustalocerus.

Ropronia pediculata. Type.-Blue label 314, yellow label 1278. 2nd Coll. Pub. Mus., Quebec. Lacks abdomen.

Roptrocerus (sic) rectus. Type.-Yellow label 1386. 2nd Coll. Pub. Mus., Quebec. Badly glued.

Sapyga maculata. Type.-Female, yellow label 754. 2nd Coll. Pub. Mus., Quebec.

Sciapteryx punctum. Type.-Yellow label 480. 2nd Coll. Pub. Mus., Quebec. Lacks hind tarsi.

Scotioneurus dives. Type.-See introduction (Aphidiinæ).

Scotioneurus stenostigma. Type.-See introduction (Aphidiinæ).

Selandria canadensis. Type.-Female, yellow label 1148; name label "Hoplocampa canadensis Prov." 2nd Coll. Pub. Mus., Quebec. Proved by Provancher Catalog.

Selandria flavicornis. Type.-Male, yellow label 60, name label "Selandria halcyon Harris." 2nd Coll, Pub. Mus., Quebec. Lacks flagellum. See introduction.

Selandria marginata. Type.-Harrington Coll. Flagellum wanting.

Selandria paupera. Type--Yellow label 696. 2nd Coll. Pub. Mus., Quebec.

Semiotellus cupræus. Type--Yellow label 920. 2nd Coll. Pub. Mus., Quebec.

Semiotellus fasciatus. Type--Yellow label 226. 2nd Coll. Pub. Mus., Quebec. Fair.

Semiotellus fuscipes. Type-Female, yellow label 918. 2nd Coll: Pub. Mus., Quebec.

Semiotellus melanicrus. Type.-Female, yellow label 627 . 2nd Coll. Pub. Mus., Quebec. Fair.

Semiotellus minimus. Type--Yellow label 921. 2nd Coll. Pub. Mus., Quebec. Fair.

Semiotellus oblongus. Type.-Female, yellow label 919. 2nd Coll. Pub. Mus., Quebec. 
LECTOTYPES OF THE SPECIES OF HYMENOPTERA (EXCEPT APOIDEA) DESCRIBED BY ABBÉ PROVANCHER.

BY A, B. GAHAN AND S. A. ROHWER, WASHINGTON, D.C.

(Continued from page 17I.)

Semiotellus suborbicularis. Type-Yellow label 972. 2nd Coll: Pub. Mus., Quebec. Fair.

Sigalphus canadensis. Type--Female, 2nd Coll. Pub. Mus., Quebec, pinned opposite male with name label and yellow label 594. Both lack head, type has wings crumpled.

Sigalphus trisectus. Type--Male, blue label 115; yellow label 1302. 2nd Coll. Pub. Mus., Quebee.

Spalangia aenea. Type--Harrington Coll.

Spathius laflammei. Type-Female, yellow label 563 . 2nd Coll. Pub. Mus., Quebec. Dirty, apices of antennæ gone:

Sphacophilus crawii. Type.-Cat. No. 1979, U. S. Nat. Mus.

Sphaerophthalma alveolata. Type-Yellow label 1685. 2nd Coll. Pub. Mus., Quebec. Tagged "Cap Rouge."

Sphaerophthalma argentipilis. Type--Male, white label 17 (in pencil); yellow label 1406. 2nd Coll. Pub. Mus., Quebec.

Sphaerophthalma unicincta. Type--Yellow label 751. 2nd Coll. Pub. Mus., Quebec.

Sphaeropyx ovalis. Type--Female, yellow label 1272. 2nd Coll. Pub. Mus., Quebec. Fair. Badly tagged.

Sphaeropyx parvus. Type.-Female, yellow label 1027. 2nd Coll. Pub. Mus., Quebec. Apices of antennæ gone.

Sphaeropyx quebecensis. Type--Female, yellow label 601. 2nd Coll. Pub. Mus., Quebec. Right antenna and apex of left, gone.

Steniola duplicata. Type--Male, yellow label 1614. 2nd Coll. Pub. Mus., Quëbec. Apex of left antenna gone.

Stilpnus appendiculatus. Type.-Not located.

Stilpnus canadensis. Type-Male, yellow label 234. 1st Coll. Pub. Mus., Quebec. Antennæ broken at apex; abdomen, right fore-wing and all legs except one front one broken off; abdomen and part of legs glued on yellow label.

Stilpnus deficiens. Type--Harrington Coll. Antennæ missing and wings dirty.

June, 1918 
Stilpnus laevis. Type.-Yellow label 702. 2nd Coll. Pub. Mus., Quebec. Antennæ (right at 7th joint, left at scape), legs on right side at coxæ, left hind leg, right fore-wing and abdomen gone.

Strongylogaster abnormis. Type.-Female, yellow label 1147. 2nd Coll. Pub. Mus., Quebec. Allotype.-Male without label.

Strongylogaster albosectus. Type--Female, yellow label 96. 1st Coll. Pub. Mus., Quebec. . Lacks right flagellum. Two specimens without labels.

Strongylogaster impressatus. Type.-Not located.

Strongylogaster luctuosus. Type--Female, Harrington Coll.

Strongylogaster pallicoxus. - Type.-Female, yellow label 1149. 2nd Coll. Pub. Mus., Quebec. Allotype-Male, Harrington Coll. Paratype.-Female, also Harrington Coll.

Strongylogaster politus. Species must be accredited to Cresson. Type.-Female, Philadelphia No. 274. Male, yellow label 498; 1st Coll. Pub. Mus., Quebec, not a type.

Strongylogaster proximus. Type--Female, Harrington Coll. Paratype.-Yellow label 1159. 2nd Coll. Pub. Mus., Quebec.

Strongylogaster soriculatus. Type.-Female, yellow label 692; name label "Strongylogaster soriculatipes .Prov." in Prov. hand. 2nd Coll. Pub. Mus., Quebec.

Synairema americana. Type.-Female, yellow label 1081. 2nd Coll. Pub. Mus., Quebec. Lacks right flagellum.

Synairema pacifica. Type.-Female, Harrington Coll. Antennæ and right fore-wing gone.

Synaldis pilicornis. Type-Female, yellow label 1165. 2nd Coll. Pub. Mus., Quebec. Ovipositor broken off.

Syntomaspis splendens. Type.-Harrington Coll. Abdomen missing.

Tapinoma boreale. Type.-Yellow label 1403. 2nd Coll. Pub. Mus., Quebec. Eight specimens on one tag. Badly glued.

Taxonus floridanus. Type-Cat. No. 13965, U. S. Nat. Mus. Paratype.-Yellow label 1540. 2nd Coll. Pub. Mus., Quebec. 
Taxonus parens. Type.-Female, Harrington Coll. Antennæ wanting.

Taxonus robustus Type-Yellow label 931. 2nd Coll. Pub. Mus., Quebec. Flagellum gone.

Telenomus rufoniger. Type.-Yellow label 1364. 2nd Coll. Pub. Mus., Quebec. Fair.

Telenomus stygicus. Type.-White label 20; yellow label 1120. 2nd Coll. Pub. Mus., Quebec Abdomen gone.

Tenthredo atrocoerulea. Type- -Not located. Probably returned to collector. (Geddes).

Tenthredo basilaris. Type-Female, yellow label 58 and name label Tenthredo signata Nort. 2nd Coll. Pub. Mus., Quebec. Proved by Prov. Catalogue.

Tenthredo cingulata. Type--Female, yellow label 59, and name label Tenthredo verticalis Say. 2nd Coll. Pub. Mus., Quebec. Proved by Prov. Catalogue.

Tenthredo decorata. Type--Not located.

Tenthredo erythromea. Type--Harrington Coll. Female. Paratype.-Yellow label 1154. 2nd Coll. Pub. Mus., Quebec.

Tenthredo jocosa. Type.-Female, yellow label 494. 2nd Coll. Pub. Mus., Quebec.

Tenthredo lineata. Type--Female, yellow label 63. 2nd Coll. Pub. Mus., Quebec. Lacks part of flagellum.

Tenthredo mellicoxa. Type.-Female, yellow label 109; white label "109 mellicoxa:" 1st Coll. Pub. Mus., Quebec.

Tenthredo montana. Type--Not located. Probably returned to collector. (Capt. Geddes.)

Tenthredo nigricostata. Type.-Female, Harrington Coll. Lacks antennæ.

Tenthredo pallicoxa. Type.-Female, yellow label 69. 2nd Coll. Pub. Mus., Quebec. Rather dirty.

Tenthredo ruficoxa Type. - Female, Harrington Coll. Right median tarsi gone, slightly dirty.

Tenthredo terminalis. Type--Male, Harrington Coll. Left flagellum gone.

Tetrastichus trisulcatus. Type--Harrington Coll. Fair. Allotype.-Yellow label 1377. 2nd Coll. Pub. Mus., Quebec. Badly glued. 
Thalessa quebecensis. Type.-Not located.

Theocolax canadensis. Type.-Yellow label 1030. 2nd Coll. Pub. Mus., Quebec. Badly glued.

Thersilochus errabundus. Type--Harrington Coll.

Thersilochus maturus. Type--Female, yellow label 1227, blue label 106 (s). 2nd Coll. Pub. Mus., Quebec.

Thersilochus micans. Type-Female, yellow label 372 . 1st Coll. Pub. Mus., Quebec. Allotype.-Male, yellow label 1040. 2nd Coll. Pub. Mus., Quebec. Right antenna at scape missing, abdomen broken off at petiole but glued on yellow label.

Thersilochus pallipes. Type.-Female, yellow label 676 . 2nd Coll. Pub. Mus., Quebec. Antennæ broken at tips.

Thyreocera læviscutum. Type.-Yellow label 1322. 2nd Coll. Pub. Mus., Quebec. Badly glued.

Thyreopus elongatus. Type.-Not located.

Thyreopus rugosopunctatus. Type.-Female, blue-green label 851(s); yellow label 1445. 2nd Coll. Pub. Mus., Quebec.

Thyreopus sinuatus. Type.-Not located. Probably returned to collector.

Tiphia minor. Type.-Not located.

Trichacius clavatus. Type.-Blue label 764; yellow label 1376. 2nd Coll. Pub. Mus., Quebec. Fair.

Trichacis auripes. Type.-Not located.

Trichiosoma taylori. Type.-Female. Lacks left antenna. Allotype-male. Ent. Branch, Dept. Agr., Ottawa. Male paratype.-Yellow label 1151. 2nd Coll. Pub. Mus., Quebec.

Trogus canadensis. Type.-Female, yellow label 241. 1st Coll. Pub. Mus., Quebec. Allotype.-Male, yellow label 194. 2nd Coll. Pub. Mus., Quebec.

Trogus quebecensis. Type-Female, yellow label 240 . 1st Coll. Pub. Mus., Quebec. Right antenna gone.

Tropistes elegans. Type.-Not in Pub. Mus., Quebec, unless under Arotes amoenus Cress.

Tryphon canaliculatus. Type.-Female, yellow label 325 . 2nd Coll. Pub. Mus., Quebec. Fair.

Tryphon clapini. Type.-Female, yellow label 421. 1st Coll. Pub. Mus., Quebec. 
Tryphon clypeatus. Type.-Male, yellow label 333. 2nd Coll. Pub. Mus., Quebec.

Tryphon dionnei. Type--Female, yellow label 657. 2nd Coll. Pub. Mus., Quebec. -Lacks left flagellum.

Tryphon dorsalis. Type.-Female, yellow label 363. 2nd Coll. Pub. Mus., Quebec.

Tryphon dufresnei. Type--Female, not located. Allotype.-Male, yellow label 329. 2nd Coll. Pub. Mus., Quebec.

Tryphon excavatus. Type--Female, yellow label 1560. 2nd Coll. Pub. Mus., Quebec. Lacks apex of right antenna.

Tryphon fractus. Type--Male, blue label 560. 2nd Coll. Pub. Mus., Quebec.

Tryphon hervieuxii. Type-Female, yellow label 656 . 2nd Coll. Pub. Mus., Quebec. Head gone.

Tryphon pediculatus. Type.-Male, yellow label 1236. 2nd Coll. Pub. Mus., Quebec.

Tryphon rufigaster. Type-Female, yellow label 1562. 2nd Coll. Pub. Mus., Quebec.

Tryphon rufopectus. Type--Male, yellow label 1563. 2nd Coll. Pub. Mus., Quebec. Poor condition, apices of flagellum gone.

Tryphon scutellaris. Type--Not in Pub. Mus., Quebec, unless as var. of dufresnei Prov.

Tryphon tuberculifer. Type.-Female, yellow label 1237; labeled "Tryphon tuberculiferus Prov." 2nd Coll. Pub. Mus., Quebec.

Trypoxylon striatum. Type.-Not located. Probably returned to collector.

Urocerus tricolor. Type.-Female, yellow label 72. 2nd Coll. Pub. Mus., Quebec.

Westwoodia fumipennis. Type.-Female, yellow label 388 . 1st Coll. Pub. Mus., Quebec.

Xiphidion canadensis. Type--Female, yellow label 138. 1st Coll. Pub. Mus., Quebec.

-Xiphydria canadensis. Type-Female, yellow label 137. 1st Coll. Pub. Mus., Quebec. Apices of left wings wanting.

Xorides canadensis. Type.-Not in Pub. Mus., Quebec, unless under Xylonomus albopictus Cress. 
Xylonomus calidus. Type.-Female, blue label 596, in large figures. 2nd Coll. Pub. Mus., Quebec.

Xylonomus lavallensis. Type.-Not in Pub. Mus., Quebec, unless under Xylonomus humeralis Say.

Zele basalis. Type.-Female, white label 81 ; yellow label 1483. 2nd Coll. Pub. Mus., Quebec. Badly tagged.

Zele cinctus. Type.-Male, yellow label 729. 2nd Coll. Pub. Mus., Quebec. Abdomen, left legs, left fore-wing and part of antennæ gone.

Zele curtus. Type.-Female, blue label 277(s); yellow label 1276. 2nd Coll. Pub. Mus., Quebec. Wings somewhat matted.

Zele gracilis. Type.-Male, yellow label 1277. 2nd Coll. Pub. Mus., Quebec. Badly tag-mounted.

Zele palliventris. Type.-Female, yellow label 573. 2nd Coll. Pub. Mus., Quebec. Lacks left flagellum and all legs except left front leg; abdomen glued on card.

Zele rufipes. Type--Female, yellow label 731. 2nd Coll. Pub. Mus., Quebec.

\section{THE EARLY STAGES OF EMPOASCA TRIFASCIATA GILL. ${ }^{1}$}

BY HARRY B. WEISS AND EDGAR L. DiCKERSON, ${ }^{*}$ NEW BRUNSWICK, N. J.

While not recorded in Smith's List of the Insects of New Jersey, this attractive little leaf-hopper appears to be fairly well distributed in New Jersey, having been found by the writers on poplar at Irvington, Red Bank, Ridgefield, Trenton and New Brunswick. De Long in his "Leafhoppers or Jassoidea of Tennessee" (Bull. 17, Vol. V, No. 2, Tenn. State Bd. Ent.) records it as being swept from cottonwood at Clarksville, Tenn., and Van Duzee in his check list of the "Hemiptera of America, North of Mexico," gives its distribution roughly as "Eastern States to the Mississippi Valley and Eastern Canada." It was described by Gillette in the Proc. U. S. Nat. Mus., vol. XX, p. 726 (1898) from specimens taken at Douglas Co., Kansas, Champaign, Ill.,

${ }^{1}$ Identified by E. P. Van Duzee,

*The arrangement of the authors' names has no significance, and indicates neither seniority nor precedence.

June, 1918 